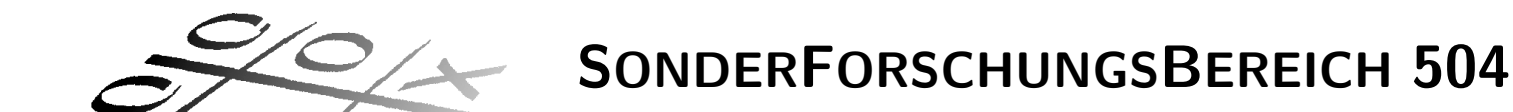

Rationalitätskonzepte,

Entscheidungsverhalten und

ökonomische Modellierung

No. $07-57$

\title{
Farsighted Coalitional Stability in TU-games
}

Sylvain Béal*

and Jacques Durieu**

and Philippe Solal ${ }^{* * *}$

August 2007

Financial support from the Deutsche Forschungsgemeinschaft, SFB 504, at the University of Mannheim, is gratefully acknowledged.

*Sonderforschungsbereich 504, email: sylvain.beal@awi.uni-heidelberg.de

${ }^{* *}$ CREUSET, University of Saint-Etienne, email:

${ }^{* * *}$ CREUSET, University of Saint-Etienne, email:

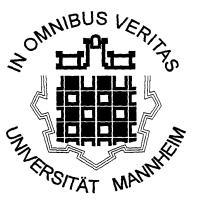

Universität Mannheim

L 13,15

68131 Mannheim 


\title{
Farsighted Coalitional Stability in TU-games ${ }^{1}$
}

SYLVAIN BÉAL ${ }^{a}$, JACQUES DURIEU ${ }^{b}$, PHILIPPE SOLAL $^{b}$

February 2007

${ }^{a}$ Alfred Weber Institut, Universität Heidelberg, 69117 Heidelberg, Germany.

${ }^{b}$ CREUSET, University of Saint-Etienne, 42023 Saint-Etienne, France.

\begin{abstract}
We study farsighted coalitional stability in the context of TUgames. Chwe $(1994$, p.318) notes that, in this context, it is difficult to prove nonemptiness of the largest consistent set. We show that every TU-game has a nonempty largest consistent set. Moreover, the proof of this result points out that each TU-game has a farsighted stable set. We go further by providing a characterization of the collection of farsighted stable sets in TU-games. We also show that the farsighted core of a TU-game is empty or is equal to the set of imputations of the game. Next, the relationships between the core and the largest consistent set are studied in superadditive TU-games and in clan games. In the last section, we explore the stability of the Shapley value. It is proved that the Shapley value of a superadditive TU-game is always a stable imputation: it is a core imputation or it constitutes a farsighted stable set. A necessary and sufficient condition for a superadditive TU-game to have the Shapley value in the largest consistent set is given.
\end{abstract}

Keywords: Clan games, Consistent set, Farsighted stable set, Shapley value.

JEL Classification Numbers: C70, C71, C72.

\section{Introduction}

The aim of this paper is to study farsighted stability proposed by Chwe (1994) in cooperative games with transferable utility. We consider the three solution

\footnotetext{
${ }^{1}$ Corresponding author: Sylvain Béal. E-mail: sylvain.beal@awi.uni-heidelberg.de

We thank Jürgen Eichberger and Anke Gerber for useful discussions. For helpful comments received, the authors also want to thank seminar participants at the workshop "Coalition Theory Network" 2007 and at the Universities of Heidelberg and CergyPontoise. Support by the German Science Foundation (DFG), through SFB 504, is gratefully acknowledged.
} 
concepts introduced by Chwe (1994) which capture foresight of the players: the farsighted core, the farsighted stable set and the largest consistent set. The core and the stable set were originally defined by Gillies (1953) and von Neumann and Morgenstern (1944) respectively and have been criticized by Harsanyi (1974) and Chwe (1994) for being too myopic. To discuss the core and the stable set, Harsanyi and Chwe envisaged a bargaining process where coalitions freely form, act publicly but cannot write binding contracts. This bargaining process specifies what each coalition can do if and when it forms without referring to the details of the procedure that determine the formation of such a coalition. The rules of this process are volontary amorphous but capture the selfishness of the players. This way of defining decision situations in cooperative games is similar to Rosenthal's "effectiveness form" (Rosenthal, 1972, Chwe, 1994). At each step of the process, a coalition of players forms and proposes a feasible imputation through that coalition, that is, an alternative outcome that the coalition is able to enforce. This proposal is accepted by that coalition if each of its members has individually a strict interest in demanding this imputation rather than accepting the imputation proposed for them at the previous step. If both conditions, feasibility and strict better response property, are satisfied, we say that the proposal made at that step directly dominates the imputation under consideration and will replace it. Then again, another coalition may propose another imputation, and so on. The core is precisely the set of imputations which are not directly dominated by any other imputation. The core does not take into account the credibility of a dominating imputation. The stable set amends the core by insisting that a dominating alternative to be credible. An important assumption underlying the direct dominance relation is that players' expectations are adaptive or myopic in the sense that at each step of the process players expect the present proposal to prevail in the next step: a coalition of players does not consider the possibility that, once it acts, another coalition might make a counter proposal, a third coalition might in turn react, and so on without limit.

To illustrate the problem of myopia, consider a bankruptcy problem with four claimants. Assume that a firm goes bankrupt leaving an estate with value 7 and four creditors or claimants with claims $d_{1}=13$, $d_{2}=9, d_{3}=21$ and $d_{4}=4$, respectively. In order to study the problem of how to divide the estate, O'Neill (1982) introduced a companion cooperative game, called the bankruptcy game, with coalition function defined as 
$v(S)=\max \left\{7-\sum_{i \notin S} d_{i}, 0\right\}$ for each coalition $S$ of claimants. Value $v(S)$ is the payoff coalition $S$ is certain to get without going to the court, i.e., either nothing or what is left after all creditors not in $S$ are paid their claims. So, $v(\{1,2,3,4\})=7, v(\{1,2,3\})=3$ and $v(S)=0$ for each other coalition of claimants. Although the coalition $\{1,2,3\}$ has a simple internal structure, this bankruptcy game has several interesting properties. Firstly, players 1, 2, 3 are veto players. Secondly, the coalition function $v$ is supermodular which implies that the core is nonempty and coincides with the unique stable set. Thirdly, the Shapley value belongs to the core. Fourthly, this bankruptcy game is a clan game. By Theorem 2.1 in Potters, Poos, Tijs, Muto (1989), the bargaining set of this game coincides with the core and the shape of the core is defined as the set of imputations for which the "small" claimant 4 can expect at most 4 (see section 4 for details). Consequently, the core contains imputations where each strict subset of veto players are forced to null payoffs. This serves to underscore the idea that myopic decisions can lead to aggressive behavior by some (veto) players that cannot be blocked by any other coalition. Because of the myopic aspect of the decisions, a coalition of players does not envisage the possibility that a proposal can be used as a message to induce further deviations that can lead to increase its final payoffs. In the strategic environments we analyze, foresight implies that a coalition of players, in making a decision, exploits all the opportunities of the game for the sake of an ultimate gain.

Consider the small claimant and assume that the status quo is the Shapley value, where he gets 1 . We claim that it is in the interest of the small claimant, being farsighted, to make a counter offer which consists in allocating 0 to each player except to the veto player 3 who will receive the maximum payoff 7. In doing so, the small claimant takes the risk of losing gain, but he anticipates that the veto players 1 and 2 will react but will be unable to improve their final payoffs without the cooperation of player 3. To be precise, given the feasibility constraints, the small claimant knows that the veto players 1 and 2 will make a proposal which in turn will induce the formation of the clan $\{1,2,3\}$. Indeed, coalition $\{1,2\}$ does not have the power to enforce an imputation which allocates a strictly positive payoff to their members. Thus, coalition $\{1,2\}$ has an incentive to make a counter offer where player 3 will receive a null payoff. Then, the veto player 3 will be forced to react but as players 1 and 2 are also veto players, he will need their cooperation to expect a strictly positive payoff. Given the feasibility constraint, if coalition 
$\{1,2,3\}$ forms, it cannot distribute to its members a total payoff superior to 3 . It follows that the small claimant will receive at least the complementary value, that is 4 , and each veto player will accept to negociate a strictly positive share of 3. Each player strictly prefers the last imputation of this sequence of moves to the imputation he faces at the step where he or she decides to join the deviating coalition. Following Chwe (1994), we say that the Shapley value is indirectly dominated through a sequence of moves of length three. In section 4, we show that in a large class of bankruptcy games with farsighted players, each farsighted stable imputation distributes to each veto player a strictly positive payoff. Moreover, if the bankruptcy game possesses only one small claimant, he or she will get on each farsighted stable imputation at least the best payoff he can expect in the core. This contrasts strongly with the payoffs that the core, the stable set, the bargaining set and the Shapley value allocate to the players in this class of bankruptcy games.

In that example, there exists an infinite number of sequences of moves which indirectly dominate the Shapley value. When it is common knowledge that the players are farsighted, we also see that each coalition on such a sequence has to specify the payoffs obtained by the players outside the coalition in order to prevent or to prompt some subsequent proposals. Part of the strategic aspect of this environment is captured by the full specification of imputations at each step of the process. One could object that such sequences of coalitions will not actually form since at each step of the process several coalitions might form and move toward some other directions. Also, would the coalitions move from the Shapley value to another imputation if the latter is not farsighted stable? But, indirect dominance is interpreted in the following way: if an imputation $z$ indirectly dominates an imputation $x$ and $z$ is presumed stable, then it is possible, not certain, that the sequence of coalitions will form and move from $x$ to $z$. That is, players think that any coalition capable of responding might be the one that responds.

Chwe's (1994) farsighted stability notion is based on the indirect dominance relation. It is applied to social environments more general than those of cooperative games considered in this paper. The intention is to define weak solution concepts which do not predict what will be played but which outcomes are eliminated with confidence. Chwe incorpored sequences of moves into the definition of the core and of a stable set. We call such sets farsighted core and farsighted stable set respectively. He defined another solution con- 
cept as a consistent set. The consistent set is similar to the farsighted stable set but differs from it in how deviating coalitions anticipate moves that might follow. In the case of farsighted stable set, deviating coalitions are optimistic and expect that later moves will terminate at their most favorable outcome. On the contrary, the consistent set assumes that defectors have pessimistic expectations. Although there can be many consistent sets, there uniquely exists a largest consistent set, that is, a consistent set which contains all others. Each farsighted stable set is contained in the largest consistent set. Within the framework of cooperative games with transferable utility, Chwe (1994, p.318) notes that the largest consistent set has two main drawbacks: proving nonemptiness beyond the class of 3-player games is difficult and all but simple examples are hard to compute. The two other solution concepts, the farsighted core and the farsighted stable set, are not fully investigated by Chwe. Diamantoudi and Xue (2005) point out that the existence of farsighted stable set in games with transferable utility remains an open question. It seems relevant to cope with the difficulties mentioned above since myopic and farsighted behaviors can lead to very different outcomes as emphasized in the foregoing bankruptcy game.

In this paper, we prove the following assertions:

1. Each cooperative game with transferable utility admits a nonempty largest consistent set.

2. Each cooperative game with transferable utility admits a nonempty farsighted stable set. There are two possibilities: the game admits a unique farsighted stable set and, in this case, it coincides with the set of imputations, or the game admits an infinite collection of singleton farsighted stable sets.

3. The farsighted core of a cooperative game with transferable utility is empty if and only if the game admits an infinite collection of singleton farsighted stable sets. If the farsighted core is nonempty, then it consists of the set of imputations of the game.

4. The Shapley value of a superadditive cooperative game with tranferable utility is always a stable imputation: it is a core imputation or it constitutes a farsighted stable set. 
The rest of the paper is organized as follows. Section 2 gives definitions and presents the solution concepts of Chwe (1994). In section 3 the above mentioned concepts are used to predict which imputations are farsighted stable in cooperative games with transferable utility. Points 1-3 are proved. We identify the class of games which admit a collection of singleton farsighted stable sets and provide a description of this collection. The properties of farsighted stable imputations are also studied. In particular, we exhibit a large class of superadditive games for which core imputations are not eliminated by farsighted players. The discussion also reveals that for the foregoing class of games, each strictly individually rational imputation belongs to the largest consistent set. In section 4, the relationships between the core and the farsighted stability solutions are investigated in clan games. We show that farsighted players eliminate all imputations which are not strictly individually rational for each veto player. One implication is that the largest consistent set cannot contain the core of a clan game. In section 5 , we study the stability of the Shapley value in superadditive games. Point 4 is proved. In addition, a necessary and sufficient condition for a superadditive game to have the Shapley value in the largest consistent set is given. Section 6 contains concluding remarks.

\section{Preliminaries}

Let $\subseteq$ denote weak set inclusion and $\subset$ denote proper set inclusion. We use the notation $|S|$ to denote the number of elements in a finite set $S$.

\section{$2.1 \quad$ TU-games}

A cooperative game with transferable utility, or simply a $T U$-game, consists of a finite set of players $N$ and a coalition function $v: 2^{N} \longrightarrow \mathbb{R}, v(\emptyset)=0$, which describes for each coalition of players $S \in 2^{N}$ the maximal wealth of transferable utility $v(S)$ that they would have to divide among themselves if they were to cooperate together and with no one outside $S$. Throughout, we assume that $|N| \geq 3$. For a coalition $S \in 2^{N}, v_{\mid S}$ denotes the restriction of the coalition function $v$ to the player set $S$, that is, $v_{\mid S}(T)=v(T)$ for each $T \in 2^{S}$. For each coalition $S \in 2^{N}$, the TU-game $\left(S, v_{\mid S}\right)$ is called a subgame of the TU-game $(N, v)$. A TU-game $(N, v)$ is said to be essential if $v(N)>\sum_{i \in N} v(\{i\})$, and inessential otherwise. A TU-game $(N, v)$ is 
superadditive if $v(S \cup T) \geq v(S)+v(T)$ for all disjoint coalitions $S, T \in$ $2^{N}$. Note that a superadditive TU-game $(N, v)$ is essential if and only if there are disjoint coalitions $S$ and $T$ such that $v(S \cup T)>v(S)+v(T)$. A superadditive inessential TU-game has necessarily an additive $v$, that is, $v(S)=\sum_{i \in S} v(\{i\})$ for all $S \in 2^{N}$. A TU-game $(N, v)$ is supermodular if $v(S \cup\{i\})-v(S) \geq v(T \cup\{i\})-v(T)$ for all $S, T \in 2^{N \backslash\{i\}}, S \supseteq T$, that is, the marginal contribution of any player $i \in N$ does not decrease when he joins a larger coalition. Note that a supermodular game is superadditive.

\subsection{Set-valued solution concepts}

The players in a TU-game $(N, v)$ are eventually interested in what they individually will get out of cooperating with other players. An allocation is a function $x$ from $N$ to $\mathbb{R}$ that specifies for each player $i \in N$ the payoff $x(i) \in$ $\mathbb{R}$ that this player can expect when he cooperates with the other players. For each allocation $x \in \mathbb{R}^{N}$, each coalition $S \in 2^{N}$, we write $x(S)=\sum_{i \in S} x(i)$. Clearly, $x(\emptyset)=0$. An allocation $x \in \mathbb{R}^{N}$ is efficient if $x(N)=v(N)$ and is individually rational if $x(i) \geq v(\{i\})$ for each player $i \in N$. An allocation $x \in \mathbb{R}^{N}$ is an imputation if it is efficient and individually rational. The set of all imputations of a TU-game $(N, v)$ is denoted by

$$
I(N, v)=\left\{x \in \mathbb{R}^{N} \mid x(N)=v(N) \text { and } x(i) \geq v(\{i\}) \text { for each } i \in N\right\} .
$$

A given imputation $x$ is called interior if it assigns a payoff $x(i)>v(\{i\})$ to each player $i \in N$. A set-valued solution concept is a rule $\Phi$ that assigns to each TU-game $(N, v)$ a collection $\Phi(N, v)$ of subsets in $I(N, v)$. If a TU-game is inessential, then the only conceivable imputation $x$ is given by $x(i)=v(\{i\})$ for each $i \in N$. In such case, $v(N)=\sum_{i \in N} v(\{i\})$. So, there is no need to study solution concepts in the latter case, and one typically restricts the analysis to the class of essential games.

The set of all imputations $I(N, v)$ of a TU-game $(N, v)$ is the set of all alternatives of the game. Each coalition has preferences among the various imputations. However, only certain of these preferences are effective in the sense that the coalition $S$, if acting together, can enforce this preference on the whole group. For any two distinct imputations $x$ and $z, x$ is called feasible from $z$ via the nonempty coalition $S$ if $v(S) \geq x(S)$. This condition means that $S$ has the power to guarantee a wealth at least as good as $x(S)$ 
and so has the power to replace $z$ by $x$. By $z \longrightarrow_{S} x$ we denote that the nonempty coalition $S$ has the power to replace the imputation $z$ by the imputation $x$. Note that $z \longrightarrow_{S} x$ if and only if $y \longrightarrow_{S} x$ for each imputation $y \in I(N, v)$. We say that a nonempty coalition $S$ effectively prefers imputation $x$ to imputation $z$ if (a) $z \longrightarrow_{S} x$ and (b) $z(i)<x(i)$ for each $i \in S$. Condition (a) says that $S$ has the power to replace $z$ by $x$, and condition (b) says that $S$ strictly prefers $x$ to $z$. We say that imputation $x$ directly dominates imputation $z$, which we write $z<x$, if there exists a nonempty coalition $S$ which effectively prefers $x$ to $z$.

The core. The core of a TU-game is the subset of all imputations $x \in I(N, v)$ that no other imputation directly dominates. We denote by $C$ the rule that assigns to each TU-game $(N, v)$ its core, that is,

$$
C(N, v)=\{x \in I(N, v) \mid \nexists z \in I(N, v) \text { such that } x<z\} .
$$

When the TU-game $(N, v)$ is superadditive, the core is characterized by the subset of all imputations that are acceptable for each coalition, that is,

$$
C(N, v)=\left\{x \in I(N, v) \mid x(S) \geq v(S) \text { for each } S \in 2^{N}\right\} .
$$

This characterization has the following interesting interpretation. It says that an imputation is in the core if and only if no coalition has an incentive to split off from the grand coalition $N$ to form a smaller coalition because they collectively receive at least as much as what they can obtain for themselves as a coalition.

Chwe (1994), among others, criticizes the core for being too myopic. Myopia is reflected by the fact that a coalition does not take into account the possibly that the rest of the players may regroup. That is, after a coalition forms to induce a feasible imputation from an initial one, another coalition and then a third coalition would form to induce new feasible imputations, and so on. Players are farsighted when they take into account such a sequence of moves and evaluate their payoffs in the end. For this reason, Chwe (1994), in the spirit of Harsanyi (1974, section Postscript, p.1494), suggests to replace the direct dominance relation by some "indirect dominance" relation, which captures the fact that farsighted players consider the final alternatives that their moves may lead to. So, a coalition may choose to replace an imputation by another one, which does not necessarily make its members strictly better 
off, as long as its move leads to final imputations that its members strictly prefer; similarly, a coalition may refuse to replace an imputation by another it effectively prefers if its move eventually leads to imputations that make at least one of its members not strictly better off. For $x, z \in I(N, v)$, we say that $x$ indirectly dominates $z$, which we write $z \ll x$, if there exist a finite sequence of imputations $z=x^{1}, x^{2}, \ldots, x^{p-1}, x^{p}=x$ and a finite sequence of nonempty coalitions $S^{1}, S^{2}, \ldots, S^{p-1}$ such that for each $j=1,2, \ldots, p-1$, (a) $x^{j} \longrightarrow_{S^{j}} x^{j+1}$ and (b) $x^{j}(i)<x(i)$ for each $i \in S^{j}$. Condition (a) says that each coalition $S^{j}$ has the power to replace imputation $x^{j}$ by imputation $x^{j+1}$, and condition (b) says that each player in $S^{j}$ strictly prefers imputation $x$ to imputation $x^{j}$. An infinite sequence of imputations $\left(x^{t}\right)_{t \in \mathbb{N}}$ is called an infinite $\ll$-chain if $t<l$ implies $x^{t} \ll x^{l}$.

It is clear from the definition of indirect dominance that $z<x$ if $p=1$. It is worth noting that in the definition of indirect dominance we assume that moves are not binding in the sense that some players belonging to a coalition at one step of the sequence may later initiate another move with players within or even outside the coalition. Players take a forward-looking attitude and compare the current imputation with the anticipated final imputation. Thus, we assume that coalitions freely form, act publicly, and that the negociation is repeated with moves and counter moves.

We can now examine three other different set-valued solution concepts which capture foresight of the players.

The farsighted core. The farsighted core of a TU-game $(N, v)$ is the subset of imputations that are not dominated with respect to $\ll$. We denote by $F C$ the rule that assigns to each TU-game $(N, v)$ its farsighted core, that is,

$$
F C(N, v)=\{x \in I(N, v) \mid \nexists z \in I(N, v) \text { such that } x \ll z\} .
$$

Obviously, $F C(N, v) \subseteq C(N, v)$. The farsighted core, like the core, does not consider the credibility of the dominating alternative; hence it is too exclusive and even more likely to be empty. The following farsighted von Neumann-Morgenstern stable sets amend the farsighted core by insisting that a dominating alternative to be credible.

The farsighted stable sets. Let $(N, v)$ be a TU-game. A subset $K$ of $I(N, v)$ is farsighted stable if (a) for all $x, z \in K$ neither $z \ll x$ nor $x \ll z$, 
and (b) for all $z \in I(N, v) \backslash K$ there exists $x \in K$ such that $z \ll x$. Conditions (a) and (b) are called internal stability and external stability, respectively. We denote by $F S$ the rule that assigns to each TU-game $(N, v)$ its collection of farsighted stable sets, that is,

$$
F S(N, v)=\{K \subseteq I(N, v) \mid K \text { is farsighted stable }\} .
$$

By external stability of the farsighted stable set and internal stability of the farsighted core, we have $F C(N, v) \subseteq K$ for each $K \in F S(N, v)$. It may be the case that $F S(N, v)=\emptyset$. The von Neumann-Morgenstern (1944) setvalued solution concept assigns to each TU-game the collection of stable sets of $(I(N, v),<)$ instead of $(I(N, v), \ll)$.

As noted by Chwe (1994), however, a farsighted stable set is too "exclusive", in that its exclusion of some alternatives may not be consistent with rationality and foresight (see examples p.310). To rectify this, Chwe suggests a new set-valued solution concept for social environments: the largest consistent set. We apply this concept to TU-games.

The largest consistent set. Let $(N, v)$ be a TU-game. A set $K \subseteq I(N, v)$ is consistent if it satisfies the following two conditions: (a) for each $x \in K$, each $z \in I(N, v)$, each $S \in 2^{N} \backslash\{\emptyset\}$ such that $x \longrightarrow_{S} z$, there exists $y \in K$, where $y=z$ or $z \ll y$, such that $y(i) \leq x(i)$ for at least one $i \in S$; (b) for each $x \in I(N, v) \backslash K$, there exist $z \in I(N, v), S \in 2^{N} \backslash\{\emptyset\}$ with $x \longrightarrow_{S} z$ such that for each $y \in K$, where $y=z$ or $z \ll y$, it holds that $x(i)<y(i)$ for each $i \in S$. Conditions (a) and (b) are called internal consistency and external consistency, respectively. The largest consistent set is the unique maximal consistent set with respect to $\subseteq$. We denote by $L C S$ the rule that assigns to each TU-game $(N, v)$ its largest consistent set.

The largest consistent set and the farsighted stable sets are interrelated. In the definition of the consistent set, if we replace "there exists" in (a) by "for each", and "for each $y \in K$ " by "there exists $y \in K$ " in (b), then we obtain the definition of the farsighted stable set. For a farsighted stable set $K$, any coalition of players making a counter offer from any imputation in $K$ is not better off in all ensuing imputations coming back to $K$, and some coalition making a counter offer from any imputation outside $K$ is better off in some ensuing imputations going to $K$. On the other hand, for the largest 
consistent set $L C S(N, v)$, any coalition of players making a counter offer from any imputation in $K$ is not better off in some ensuing imputations coming back to $\operatorname{LCS}(N, v)$, and some coalition making a counter offer from any imputation outside $\operatorname{LSC}(N, v)$ is better off in all ensuing imputations going to $\operatorname{LSC}(N, v)$. These solution concepts are different in the prospect of each player for the others' behavior following her deviation. Thus for a farsighted stable set, each coalition of players has an optimistic prospect and for the largest consistent set, it has a pessimistic prospect. This remark suggests that the largest consistent set contains each farsighted stable set (see Chwe, 1994, Proposition 3), that is, for each TU-game $(N, v)$, each $K \in F S(N, v)$, it holds that $K \subseteq \operatorname{LCS}(N, v)$. Since each farsighted stable set contains the farsighted core, it also holds that $F C(N, v) \subseteq \operatorname{LCS}(N, v)$. Hence, to prove nonemptiness of the largest consistent set in essential TU-games, it is sufficient to show that $F C(N, v) \neq \emptyset$. Unfortunately, we cannot proceed this way because it is well known that the core, and thus the farsighted core, of an essential TU-game may be empty. In order to prove that each essential TU-game has a nonempty largest consistent set, we will show that it admits a farsighted stable set.

\section{$3 \quad$ Farsighted stability}

The first Theorem of this section states that each essential TU-game possesses a nonempty largest consistent set:

Theorem 1 The set-valued solution concept $L C S$ satisfies nonemptiness on the class of essential TU-games: $\operatorname{LCS}(N, v) \neq \emptyset$ for each essential TU-game $(N, v)$.

Chwe (1994, Proposition 2) gives a sufficient condition under which the largest consistent set is nonempty in social environments. But his result applies only to social environments where the set of alternatives from which players' preference relations are defined is denumerable. Since in essential TU-games the set of imputations is not denumerable, Chwe's nonemptiness result cannot be used to prove Theorem 1. Xue (1997) extends Chwe's nonemptiness result of the largest consistent set by relaxing the condition of denumerability of the set of alternatives. Part of Xue's result will be useful to construct our proof. In the context of TU-games, Xue's nonemptiness result can be stated as follows: 
Theorem 2 (Xue, 1997)

Let $(N, v)$ be a TU-game. Assume that if there exists an infinite «-chain, there exists $x \in I(N, v)$ such that $z \ll x$ for each $z \in I(N, v) \backslash\{x\}$. Then, $\operatorname{LCS}(N, v) \neq \emptyset$.

Xue's Theorem provides a sufficient condition for nonemptiness of the largest consistent set when an infinite «-chain exists. In other cases, the largest consistent set is nonempty. Below, we show that the class of essential TUgames admits elements that contain infinite «-chains. In fact, it turns out that essential TU-games satisfy the sufficient condition of Xue's Theorem. Before proceeding to the proofs of these claims, we need the following definitions. For each TU-game $(N, v)$ define $E(N, v)$ as the set of coalitions $S,|S| \geq 2$, such that $v(S)>\sum_{i \in S} v(\{i\})$. So, a TU-game $(N, v)$ is essential if $N \in E(N, v)$. In what follows, for each essential TU-game $(N, v)$, denote by $P(N, v)$ the set of ordered pairs $(x, S)$, where $x \in I(N, v)$ and $S \in E(N, v) \backslash\{N\}$, such that $x(S) \leq v(S)$ and $x(i)>v(\{i\})$ for each $i \in S$. The following two Facts will be useful throughout the paper.

Fact 1 Let $(N, v)$ be a TU-game such that $E(N, v) \supset\{N\}$. Then, $P(N, v) \neq$ $\emptyset$.

Proof. Suppose that $(N, v)$ is such that $E(N, v) \supset\{N\}$. Pick any coalition $S \in E(N, v) \backslash\{N\}$ and $\varepsilon$ in $] 0, m]$, where $m$ is the minimum between $v(S)-$ $\sum_{i \in S} v(\{i\})$ and $v(N)-\sum_{i \in N} v(\{i\})$. Define allocation $x \in \mathbb{R}^{N}$ as follows: $x(i)=v(\{i\})+\varepsilon /|S|$ for each $i \in S$, and $x(i)=v(\{i\})+\left[v(N)-\sum_{i \in N} v(\{i\})-\right.$ $\varepsilon] /(|N|-|S|)$ for each $i \in N \backslash S$. We see that $(x, S) \in P(N, v)$.

Fact 2 Assume that $x$ and $z$ are two distinct imputations for an essential TU-game $(N, v)$. If $z \ll x$, then there exists a coalition $S \in 2^{N} \backslash\{\emptyset, N\}$ such that $(x, S) \in P(N, v)$.

Proof. Let $(N, v)$ be any essential TU-game. Pick any two distinct imputations $x$ and $z$ and assume that $z \ll x$. By definition of indirect dominance, there exist a finite sequence of imputations $z=x^{1}, x^{2}, \ldots, x^{p-1}, x^{p}=x$ and a finite sequence of nonempty coalitions $S^{1}, S^{2}, \ldots, S^{p-1}$ such that (a) $x^{j} \longrightarrow_{S^{j}} x^{j+1}$, for each $j=1,2, \ldots, p-1$, and (b) $x^{j}(i)<x(i)$, for each $i \in S^{j}$ for each $j=1,2, \ldots, p-1$. Consider the last move $x^{p-1} \longrightarrow S_{S^{p-1}} x$. We have, $x\left(S^{p-1}\right) \leq v\left(S^{p-1}\right)$ and $x^{p-1}(i)<x(i)$ for each $i \in S^{p-1}$. Because $x^{p-1} \in I(N, v)$, we also have $v(\{i\}) \leq x^{p-1}(i)$ for each $i \in N$ so that 
$v(\{i\})<x(i)$ for each player $i \in S^{p-1}$. It follows that $S^{p-1} \in E(N, v)$ and $\left(x, S^{p-1}\right) \in P(N, v)$.

Lemma 1 Let $(N, v)$ be a TU-game such that $E(N, v) \supset\{N\}$. Then, $(N, v)$ admits an infinite «-chain.

PROOF. Pick any essential TU-game $(N, v)$ as hypothesized and choose any pair $(x, S) \in P(N, v)$, which is possible by Fact 1 . Pick any player $j \in S$. Let $e(x,\{j\})=x(j)-v(\{j\})$ and let $\left(\varepsilon^{t}\right)_{t \in \mathbb{N}}$ be a strictly increasing sequence in $] 0, e(x,\{j\})\left[\right.$. Construct an infinite sequence of imputations $\left(x^{t}\right)_{t \in \mathbb{N}}$ as follows: for each $t \in \mathbb{N}, x^{t}(j)=x(j)-\varepsilon^{t}, x^{t}(i)=x(i)+\varepsilon^{t} /(|S|-1)$ for each $i \in S \backslash\{j\}$, and $x^{t}(i)=x(i)$ for each $i \in N \backslash S$. Define also imputation $z$ as follows: $z(i)=v(\{i\})$ for each $i \in S$ and $z(i)=v(\{i\})+(v(N)-$ $\left.\sum_{i \in N} v\{i\}\right) /(|N|-|S|)$ for each $i \in N \backslash S$. Pick any $k \in S \backslash\{j\}$. Because, $z(k)=v(\{k\})$ and $x^{l}(S) \leq v(S)$ for every $l \in \mathbb{N}, z$ is feasible from $x^{t}$ via $\{k\}$ and $x^{l}$ is feasible from $z$ via $S$, respectively. Thus, consider the moves $x^{t} \longrightarrow\{k\} z$ and $z \longrightarrow_{S} x^{l}$ for each $t, l \in \mathbb{N}$ such that $t<l$. To show: $x^{t} \ll x^{l}$. By construction, $x^{t}(k)<x^{l}(k)$ whenever $t<l$. By definition of $x$ and construction of the sequence $\left(x^{t}\right)_{t \in \mathbb{N}}$ and of $z, v(\{i\})=z(i)<x(i)$ for each $i \in S$ implies $z(i)<x^{t}(i)$ for each $i \in S$ and each $t \in \mathbb{N}$. Therefore, $t<l$ implies $x^{t} \ll x^{l}$ as desired.

Lemma 2 Let $(N, v)$ be a $T U$-game such that $E(N, v) \supset\{N\}$. Then, for each pair $(x, S) \in P(N, v)$, it holds that $z \ll x$ for each $z \in I(N, v) \backslash\{x\}$.

PROOF. Pick any TU-game $(N, v)$ as hypothesized. Pick any $z \in I(N, v) \backslash\{x\}$. Since $x(N)=v(N)=z(N)$, there is $j \in N$ such that $z(j)<x(j)$. Because $S$ contains at least two elements, we can choose $i_{1} \in S$ such that $i_{1} \neq j$. Consider such a player $i_{1}$ and define imputation $y^{1}$ as follows: $y^{1}\left(i_{1}\right)=v(N)-\sum_{i \in N \backslash\left\{i_{1}\right\}} v(\{i\})$ and $y^{1}(i)=v(\{i\})$ for each $i \in N \backslash\left\{i_{1}\right\}$. Consider any player $i_{2} \in N \backslash S$ and define imputation $y^{2}$ as follows: $y^{2}\left(i_{2}\right)=$ $v(N)-\sum_{i \in N \backslash\left\{i_{2}\right\}} v(\{i\})$ and $y^{2}(i)=v(\{i\})$ for each $i \in N \backslash\left\{i_{2}\right\}$. Choose any $i_{3} \in S \backslash\left\{i_{1}\right\}$, where $i_{3}$ is equal to $j$ in case $S=\left\{i_{1}, j\right\}$. Because $y^{1}(j)=v(\{j\}), y^{2}\left(i_{3}\right)=v\left(\left\{i_{3}\right\}\right)$, and $x(S) \leq v(S), y^{1}$ is feasible from $z$ via $\{j\}, y^{2}$ is feasible from $y^{1}$ via $\left\{i_{3}\right\}$, and $x$ is feasible from $y^{2}$ via $S$, respectively. Thus, consider the successive moves $z \longrightarrow\{j\} y^{1}, y^{1} \longrightarrow_{\left\{i_{3}\right\}} y^{2}$, and $y^{2} \longrightarrow_{S} x$. By construction, $z(j)<x(j), y^{1}\left(i_{3}\right)<x\left(i_{3}\right)$ and $y^{2}(i)<x(i)$ for each $i \in S$. Therefore, $z \ll x$. 
Proof of TheOrem 1. Pick any essential TU-game $(N, v)$. We distinguish two cases.

(a) The TU-game $(N, v)$ is such that $E(N, v)=\{N\}$. Pick any two distinct elements $x$ and $z$ in $I(N, v)$. By Fact 2 , if $z \ll x$, then there exists a coalition $S \in 2^{N} \backslash\{\emptyset, N\}$ such $(x, S) \in P(N, v)$. On the other hand, $E(N, v)=\{N\}$ implies that $P(N, v)=\emptyset$. Thus, $z \nless x$, and $x \nless z$ by the same reasoning. As $x$ and $z$ are arbitrary, there does not exist any imputation that indirectly dominates another imputation. Therefore, $I(N, v)=\operatorname{LCS}(N, v)=C(N, v)$, where the last equality follows from the fact that the direct dominance relation is a special case of the indirect dominance relation.

(b) The TU-game $(N, v)$ is such that $E(N, v) \supset\{N\}$. By Lemma $1,(N, v)$ admits an infinite «-chain. By Lemma 2, there exists $x \in I(N, v)$ such that $z \ll x$ for each $z \in I(N, v) \backslash\{x\}$. By Xue's nonemptiness Theorem, $\operatorname{LCS}(N, v) \neq \emptyset$. To see this, it suffices to note that $z \ll x$ for each $z \in I(N, v) \backslash\{x\}$ means that $\{x\}$ is externally stable. Because $x \nless x$ by definition of player's preferences, $\{x\}$ is also internally stable. Thus, $\{x\} \in F S(N, v)$. Since each farsighted stable set is included in the largest consistent, the result follows.

In part (a) of the proof of Theorem $1, I(N, v)$ constitutes trivially the unique farsighted stable set. Combining this remark with part (b) of the proof of Theorem 1, we obtain that the rule FS satisfies nonemptiness on the class of essential TU-games, that is, $F S(N, v) \neq \emptyset$ for each essential TU-game $(N, v)$. We can say more about the structure of the collection of farsighted stable sets when $E(N, v) \supset\{N\}$. More precisely, the following result shows that the collection of farsighted stable sets for an essential TU-game is either equal to the set of imputations or is a collection of singleton sets.

Theorem 3 Let $(N, v)$ be a $T U$-game. If $E(N, v) \supset\{N\}$, then

$$
F S(N, v)=\left\{\{x\} \mid \exists S \in 2^{N} \backslash\{\emptyset, N\} \text { such that }(x, S) \in P(N, v)\right\} .
$$

Otherwise, $F S(N, v)=\{I(N, v)\}$.

Proof. Suppose that $E(N, v) \supset\{N\}$. First, we show that a singleton set $\{x\}$ is farsighted stable if and only if $(x, S) \in P(N, v)$ for some coalition $S \in E(N, v) \backslash\{N\}$. Next, we show that no other subset of imputations is farsighted stable.

Pick any $x \in I(N, v)$. Assume that $(x, S) \notin P(N, v)$ for every coalition 
$S \in E(N, v) \backslash\{N\}$. If $\{x\}$ is farsighted stable, then $z \ll x$ for each $z \in$ $I(N, v) \backslash\{x\}$ by external stability. By Fact 2, if $x$ indirectly dominates an imputation $z \neq x$, then there is a coalition $S \in 2^{N} \backslash\{N, \emptyset\}$ such that $(x, S) \in P(N, v)$. It follows that $\{x\}$ cannot be farsighted stable. So, assume that there exists a coalition $S \in 2^{N} \backslash\{N, \emptyset\}$ such that $(x, S) \in P(N, v)$. By Lemma 2 and part (b) of the proof of Theorem 1, the singleton set $\{x\}$ is farsighted stable. This completes the first part of the proof.

Now, assume, by way of contradiction, that there exists a subset $K$ of imputations such that $|K| \geq 2$ and $K \in F S(N, v)$. Such a subset $K$ is necessarily different from $I(N, v)$. To see this, note that if $K=I(N, v)$, then internal stability is violated by an application of Lemma 2. So, pick any $y \in I(N, v) \backslash K$. By external stabilility, there exists $x \in K$ such that $y \ll x$. Therefore, there exists a coalition $S \in E(N, v) \backslash\{N\}$ such that $(x, S) \in P(N, v)$ by Fact 2 . By Lemma $2, z \ll x$ for all $z \in I(N, v) \backslash\{x\}$. In particular, $x$ indirectly dominates every $z \in K \backslash\{x\}$, which contradicts the internal stability of $K$. Hence, our initial assumption is false. The first part of Theorem 3 follows. The second part of the Theorem follows directly from part (a) of the proof of Theorem 1.

Remark The farsighted core of a TU-game with a nonempty core may be empty. To see this, consider the game $(N, v)$ given by $N=\{1,2,3,4\}$ and $v(S)=|S|^{2}$ for each $S \in 2^{N}$. Because this TU-game is supermodular, it has a nonempty core. Consider the following imputation $x \in C(N, v)$ given by $x(1)=x(2)=2$ and $x(3)=x(4)=6$. We have $x(\{1,2\})=v(\{1,2\})$, $x(1)>v(\{1\})$ and $x(2)>v(\{2\})$. So, $(x,\{1,2\}) \in P(N, v)$. An application of Lemma 2 yields $z \ll x$ for every $z \in I(N, v) \backslash\{x\}$, which also means that $\{x\}$ is a singleton farsighted stable set. By symmetry of $(N, v)$, the core is also symmetric and so imputation $y$ defined as $y(1)=y(2)=6, y(3)=y(4)=2$ belongs to the core of $(N, v)$. Again, $(y,\{3,4\}) \in P(N, v)$ and $\{y\}$ constitutes a farsighted stable set. We have proven that there do not exist imputations that are not indirectly dominated. The assertion follows.

This remark can be generalized in order to obtain the following result:

Theorem 4 Let $(N, v)$ be any TU-game. Then $F C(N, v)=\emptyset$ if and only if $E(N, v) \supset\{N\}$.

Proof. $(\Longrightarrow)$ Assume that $(N, v)$ is such that $E(N, v) \supset\{N\}$. By Fact 1 , we can pick $(x, S) \in P(N, v)$ such that $x(S)=v(S)$ and $x(i)>v(\{i\})$ 
for each $i \in S$. By Lemma 2 and part (b) of the proof of Theorem 1, the singleton set $\{x\}$ is farsighted stable, that is, $y \ll x$ for each imputation $y$, $y \neq x$. This implies that $\{x\} \supseteq F C(N, v)$. Because $|S| \geq 2$ and $S \neq N$, we can choose $j \in S$ and $k \in N \backslash S$. From imputation $x$, contruct allocation $z$ as follows: pick any $\varepsilon \in] 0, e(x,\{j\})[$ and set $z(j)=x(j)-\varepsilon, z(k)=x(k)+\varepsilon$, and $z(i)=x(i)$ for each $N \backslash\{j, k\}$. Then, the pair $(z, S)$ belongs to $P(N, v)$. Therefore, the singleton set $\{z\}$ is also farsighted stable by Lemma 2 and part (b) of the proof of Theorem 1, that is $y \ll z$ for each imputation $y$, $y \neq z$. In particular, $x \ll z$. Hence, $x$ cannot belong to the farsighted core of $(N, v)$, and so, $F C(N, v)=\emptyset$.

$(\Longleftarrow)$ By contraposition. Assume that the TU-game $(N, v)$ is such that $E(N, v)=\{N\}$. To show: $F C(N, v) \neq \emptyset$. By part (a) of the proof of Theorem 1, we know that $I(N, v)=C(N, v)$ so that no imputation can be indirectly dominated. It follows that $F C(N, v)=C(N, v)$.

Theorem 4 says that the farsighted core of an essential TU-game is either empty or equal to the set of imputations and thus is not very useful. Combining Theorem 3 with Theorem 4, we obtain that an essential TUgame contains at least two farsighted stable (singleton) sets if and only if its farsighted core is empty. Part (a) of the proof of Theorem 1 suggests that the largest consistent set of a TU-game contains its core. This assertion is partially true in superadditive games. The next Proposition gives a sufficient condition for a superadditive TU-games to have the core included in the largest consistent set.

Proposition 1 Let $(N, v)$ be a superadditive TU-game. Assume that if $E(N, v) \supset\{N\}$, it holds that $\bigcap_{S \in E(N, v)} S=\emptyset$. Then, $C(N, v) \subseteq L C S(N, v)$. PROOF. See appendix A.

The sufficient condition of Proposition 1 is not very restrictive. It is satisfied in strictly superadditive TU-games, that is, in TU-games $(N, v)$ where $v(S \cup T)>v(S)+v(T)$ for all disjoint coalitions $S, T \in 2^{N} \backslash\{\emptyset\}$. Note also that for the class of positive 0-normalized TU-games, this condition amounts to the absence of veto player, i.e., a player $i$ for which $v(S)=0$ for each $S$ with $i \notin S$. The following example shows that if a superadditive TU-game does not satisfy the sufficient condition of Proposition 1, the core is not necessarily included in the largest consistent set. 


\section{Example 1}

Consider the superadditive TU-game with player set $N=\{1,2,3,4\}$ and coalition function $v$ given by $v(N)=v(\{1,2,4\})=v(\{1,2,3\})=2, v(\{1,2\})=$ 1 and $v(S)=0$ otherwise. The sufficient condition of Proposition 1 does not hold because $E(N, v)=\{N,\{1,2\},\{1,2,3\},\{1,2,4\}\}$ and $\bigcap_{S \in E(N, v)} S=$ $\{1,2\}$. Pick $x$ and $x^{1}$ in $I(N, v)$, where $x$ and $x^{1}$ are defined as $x(1)=$ $x(3)=x(4)=0, x(2)=2$ and $x^{1}(1)=x^{1}(2)=0, x^{1}(3)=x^{1}(4)=1$, respectively. Imputation $x \in C(N, v)$ and imputation $x^{1} \notin C(N, v)$. Moreover, there is no coalition $S$ such that $\left(x^{1}, S\right)$ or $(x, S)$ is in $P(N, v)$. We assert that $x \notin L C S(N, v)$. To show: there exist $z \in I(N, v), S \in 2^{N} \backslash\{\emptyset\}$ with $x \longrightarrow_{S} z$ such that for each $y \in L C S(N, v)$, where $y=z$ or $z \ll y$, it holds that $x(i)<y(i)$ for each $i \in S$. Consider the move $x \longrightarrow\{1\} x^{1}$, where $x^{1}(1)=v(\{1\})$. Pick any $y \in \operatorname{LCS}(N, v)$. First, assume that $x^{1} \neq y$ and $x^{1} \ll y$. By Fact 2, there exists a coalition $S \in E(N, v) \backslash\{N\}$ such that $(y, S) \in P(N, v)$. Because player 1 belongs to $\bigcap_{S \in E(N, v)} S$, we necessarily have $v(\{1\})<y(1)$. On the other hand, $x(1)=v(\{1\})$ implies that $x(1)<y(1)$, as desired. Now, it remains to show that the case $x^{1}=y$ is not possible, that is, $x^{1} \notin L C S(N, v)$. Pick $w \in I(N, v)$ defined as $w(1)=w(2)=1 / 2, w(3)=0, w(4)=1$. Consider the move $x^{1} \longrightarrow\{1,2\} w$. We have, $x^{1}(1)<w(1)$ and $x^{1}(2)<w(2)$. Observe also that $\{1,2\}$ belongs to $\bigcap_{S \in E(N, v)} S$. Thus, by using the same reasoning as above, we can show that if $w \ll z$ for some $z \in I(N, v) \backslash\{w\}$, then $x^{1}(1)<z(1)$ and $x^{1}(2)<z(2)$. It follows that $x^{1}$ cannot belong to $\operatorname{LCS}(N, v)$, The result follows.

In the general context of social environments as defined by Chwe (1994), it has been noted that a farsighted stable set can be too exclusive while the largest consistent set can be too inclusive (see Chwe, 1994, Xue, 1998, Xue, Diamantoudi, 2003, Mauleon, Vannetelbosch, 2004). Proposition 2 below picks out a minimal set of properties on $(N, v)$ for which the largest consistent is too inclusive in the sense that it does not exclude any imputation. To understand why the largest consistent set can be too inclusive, recall that players have a pessimistic view on the part of a deviating coalition. Indeed, we can see from the definition of the largest consistent set that for each element of this set, each deviating coalition expects that ensuing deviations would terminate at the least favorable outcome for that coalition in the set at which ensuing deviations may terminate. 
Proposition 2 Let $(N, v)$ be a strictly superadditive $T U$-game with at least four players. Then, $\operatorname{LCS}(N, v)=I(N, v)$.

Proof. Assume that $(N, v)$ is strictly superadditive. We have to show that $I(N, v)$ is internally consistent. Pick $x$ and $z$ in $I(N, v)$ and $S \in 2^{N} \backslash\{\emptyset\}$ such that $x \longrightarrow_{S} z$. If $S=N$, then the argument runs as follows: by Fact 1 and Theorem 3, there is $y \in I(N, v)$ such that $z \ll y$. Because $x$ and $y$ are two imputations, there is at least one player $i \in N$ such that $y(i) \leq x(i)$. In case $S \neq N$, consider any player $j \in S$. Because the game is strictly superadditive, each non-singleton coalition $S$ belongs to $E(N, v)$. Thus, we can choose $T \in E(N, v)$ such that $|T|=2$ and $j \notin T$. Now, define imputation $y$ as $y(j)=v(\{j\}), y(i)=v(\{i\})+\left[v(T)-\sum_{i \in T} v(\{i\})\right] /|T|$ for each $i \in T, y(i)=v(\{i\})+\left[v(N)-v(T)-\sum_{i \in N \backslash T} v(\{i\})\right] /(|N|-|T|-1)$ for each $i \in N \backslash(T \cup\{j\})$. Imputation $y$ is well defined since $|N|-|T|>1$ by assumption. Observe that $(y, T) \in P(N, v)$ sot that using Theorem 3 yields $z \ll y$ and $y \in L C S(N, v)$. In addition, $j \in S$ and $y(j)=v(\{j\}) \leq x(j)$ imply that coalition $S$ does not strictly prefer $y$ to $x$. Since $x \longrightarrow_{S} z$ was an arbitrary element in the set of effective moves, Proposition 2 follows.

When a TU-game satisfies the sufficient condition of Proposition 1, it is not clear whether or not the largest consistent set coincides with the set of imputations. Proposition 3 and Example 2 below answer this question by stating that the largest consistent set contains each interior imputation, that is, all imputations which are strictly individually rational for each player. More precisely, Example 2 shows that we cannot conclude from Proposition 3 that the largest consistent set of a superadditive TU-game satisfying the sufficient condition of Proposition 1 is equal to the set of imputations.

Lemma 3 Let $(N, v)$ be a superadditive TU-game. Then, for each interior imputation $x$, it holds that $x \in C(N, v)$ or $\{x\} \in F S(N, v)$.

Proof. Choose any superadditive TU-game $(N, v)$. If $N$ is the only coalition in $E(N, v)$, then the assertion holds because $C(N, v)=I(N, v)$ by part (a) of the proof of Theorem 1 . Otherwise, pick any interior imputation $x$, that is, $x(i)>v(\{i\})$ for each $i \in N$. Assume that $x \notin C(N, v)$. Then, there exists a coalition $S \in 2^{N} \backslash\{N, \emptyset\}$ such that $x(S)<v(S)$. It then follows that $(x, S) \in P(N, v)$ and so $\{x\} \in F S(N, v)$ by Theorem 3 . 
Proposition 3 Assume that the superadditive TU-game $(N, v)$ satisfies the sufficient condition of Proposition 1. Then, each interior imputation belongs to the largest consistent set of $(N, v)$.

PROOF. Follows directly from Proposition 1 and Lemma 3.

\section{Example 2}

Consider the 4-player superadditive TU-game where $N=\{1,2,3,4\}, v(S)=$ 0 for each $S$ such that $|S|<3, v(S)=5$ for each $S$ of size 3 and $v(N)=7$. Then, $E(N, v)=\left\{S \in 2^{N}|| S \mid \geq 3\right\}$ so that $\bigcap_{S \in E(N, v)} S=\emptyset$. Consider now imputations $x$ and $z$ given by: $x(1)=x(2)=x(3)=0$ and $x(4)=7$; $z(1)=z(2)=z(3)=1$ and $z(4)=4$. We see that imputation $x$ is not interior. We are going to show that $x$ does not belong to the largest consistent set. Consider the move $x \longrightarrow\{1,2,3\} z$. By Proposition $3, z$ belongs to the largest consistent set but $x(i)<z(i)$ for each $i \in\{1,2,3\}$, as desired. Next, for each imputation $y \in L C S(N, v)$, where $z \ll y$, there exists $S \in 2^{N} \backslash\{\emptyset, N\}$ such that $(y, S) \in P(N, v)$ and $S$ is the set of players who deviate in the last step of the sequence of moves by Fact 2. By definition of $E(N, v) \backslash\{N\}$, coalition $S$ is of size 3 and so $y(S) \leq 5$. Because $y$ indirectly dominates $z$, it must be that $1 \leq z(i)<y(i)$ for each $i \in S$. Thus, $y(S) \leq 5$ and $1 \leq z(i)<y(i)$ for each $i \in S$ imply that $S=\{1,2,3\}$. It follows that if $z \ll y$, then $x(i)<y(i)$ for each $i \in S=\{1,2,3\}$, as desired.

The bankruptcy game given in introduction is a superadditive game with a clan. This game does not satisfy the sufficient condition of Proposition 1. Thus, we cannot conclude from Proposition 3 that its largest consistent set contains each interior imputation. However, we will show in the next section that the largest consistent set of this game is a strict subset of the set of interior imputations. This fact is of some interest because it implies that each veto player will get a strictly positive payoff on each farsighted stable imputation, which is not the case for all core imputations. The next section explores the properties of the farsighted stable sets and of the largest consistent set in clan games.

\section{Clan games}

The class of clan games was introduced by Potters, Poos, Tijs and Muto (1989) to model social interaction between a "powerful" coalition of players 
(the clan) and "powerless" players (nonclan members). Economic applications of such games include bankruptcy problems, production economies, information acquisition and holding situations. Potters, Poos, Tijs and Muto (1989) have shown that solution concepts on this class of TU-games have very appealing properties; we mention here, and below, that the core and the bargaining set (Aumann, Maschler, 1964) of a clan game coincide, the kernel (Davis, Maschler, 1965) consists of one imputation - the nucleolus (Schmeidler, 1969) of the game.

A TU-game $(N, v)$ is a clan game with clan $C \in 2^{N} \backslash\{N, \emptyset\}$ if it satisfies the following four properties:

Positivity: $v(S) \geq 0$ for each $S \in 2^{N}$.

Positive marginal contribution to $N: v(N)-v(N \backslash\{i\}) \geq 0$ for each $i \in N$.

Clan property: each player $i \in C$ is a veto player, that is, $v(S)=0$ for each $S \nsupseteq C$.

Union property: $v(N)-v(S) \geq \sum_{i \in N \backslash S}(v(N)-v(N \backslash\{i\}))$ if $C \subseteq S$.

Note that in a clan game the grand coalition $N$ maximizes $v$ over $2^{N}$. Every clan game with at most four players is superadditive but need not be supermodular when the number of players is equal to or greater than five. A TU-game $(N, v)$ is called a big clan game if it is a clan game where the clan contains all but one player. Big clan games can be opposed to big boss games studied in Muto, Nakayama, Potters, Tijs (1988). Big boss games are monotonic clan games where the clan consists of one player - the big boss. One of the most appealing economic applications of clan games is the bankruptcy problem. A bankruptcy problem is a tuple $B=\left(N, e,\left(d_{i}\right)_{i \in N}\right)$. A firm goes bankrupt leaving an estate with value $e>0$ and a finite set of creditors or claimants $N$ with claims $\left(d_{i}\right)_{i \in N}$, where $\sum_{i \in N} d_{i}>e$. The bankruptcy problem deals with the problem of how to divide the estate among all creditors. In order to study this problem, O'Neill (1982) proposed the bankruptcy game $\left(N, v_{B}\right)$ where $N$ is the set of creditors and $v_{B}$ is the coalition function that gives what a coalition $S$ can get for sure without going to court; i.e., by accepting either nothing, or what is left of the estate $e$ after each member $i$ 
of the complementary coalition $N \backslash S$ is paid his complete claim $d_{i}$. Hence

$$
v_{B}(S)=\max \left\{e-\sum_{i \in N \backslash S} d_{i}, 0\right\} .
$$

It is known that $\left(N, v_{B}\right)$ is a supermodular TU-game. It is also known that for each clan game, there exists a corresponding bankruptcy problem and so a corresponding bankruptcy game (Potters, Poos, Tijs and Muto, 1989, p.292). The relationships between clan games and supermodular games are studied in Branzei, Dimitrov and Tijs (2006). The following Theorem, from Potters, Poos, Tijs and Muto (1989), establishes that the core and the bargaining set of a clan game coincide and provides an explicit description of the core.

Theorem 5 (Potters, Poos, Tijs and Muto, 1989, Theorem 2.1)

Let $(N, v)$ be a clan game with clan $C$. Then, the bargaining set and the core coincide. Moreover, the core is given by

$$
C(N, v)=\{x \in I(N, v) \mid x(i) \leq v(N)-v(N \backslash\{i\}) \text { for each } i \in N \backslash C\} .
$$

Theorem 5 does not exclude core imputations which assign to some members of the clan a null payoff though the wealth that the clan earns is strictly positive (see Example 3 below). This situation is counter-intuitive since Clan property indicates that no positive wealth can be attained without the cooperation of each clan member. When we amend the myopic behavior, on the part of players, embedded in the core, this situation disappears in many instances. More precisely, when the clan $C$ belongs to $E(N, v)$, each imputation belonging to the largest consistent set assigns to each clan member a strictly positive payoff and the largest consistent set excludes some core imputations. These results are contained in the following Theorem: ${ }^{2}$

Theorem 6 Let $(N, v)$ be a clan game with clan $C \in E(N, v)$. Then, (a) for each imputation $x$ where $x(i)=0$ for some $i \in C$, it holds that $x \notin$ $\operatorname{LCS}(N, v)$, (b) $C(N, v) \nsubseteq L C S(N, v)$.

\footnotetext{
${ }^{2}$ Note that points (a) and (b) in Theorem 6 hold for a broader class of games than the class of clan games since the proof of the result only uses the Clan property. However, we state the result for clan games since the bargaining set, which incorporates foresight in the behavior of the players, coincide with the core for that class of games.
} 
Proof. (a) Pick any clan game $(N, v)$ with clan $C \in E(N, v)$. We show that there does not exist $x \in \operatorname{LCS}(N, v)$ which assigns to some members of the clan a null payoff. Because $C \in E(N, v),|C| \geq 2$. By definition of the clan game, it holds that $v(\{i\})=0$ for each $i \in N, v(C)>0$, and $C=\bigcap_{S \in E(N, v)} S$. For each nonempty coalition $S \subseteq C$, define imputations $x^{S}$ as follows: $x^{S}(S)=0$ and $x^{S}(N \backslash S)=v(N)$. First, we show that imputations $x^{C}$ do not belong to the largest consistent set. To this end, define imputation $z$ as follows: $z(i)=v(C) /|C|$ for each $i \in C$ and $z(i)=[v(N)-v(C)] /(|N|-|C|)$ for each $i \in N \backslash C$. Consider any move $x^{C} \longrightarrow_{C} z$, where $z(C)=v(C)$. It holds that $(z, C) \in P(N, v)$. Then, the singleton set $\{z\}$ is farsighted stable by Theorem 3 and so $z$ belongs to the largest consistent set. But, $x^{C}(i)<z(i)$ for each $i \in C$. Now, consider any $y \in \operatorname{LCS}(N, v)$, where $z \ll y$. By Fact 2 , there must exist $T \in E(N, v) \backslash\{N\}$ such that $(y, T) \in P(N, v)$. This implies that $T \supseteq C$ and so $x^{C}(i)=v(\{i\})<y(i)$ for each $i \in C$. We conclude that every $x^{C}$ satisfies condition (b) of the definition of the largest consistent set which means that $x^{C} \notin L C S(N, v)$. To show that for each $S \subset C$ and each $x^{S}$, imputation $x^{S} \notin L C S(N, v)$, we proceed as follows: consider any move $x^{S} \longrightarrow_{S} x^{C}$, where $x^{S}(S)=x^{C}(S)=0$. Using the same reasoning as above, $S \subset C$ implies that $S \subset T$ for each $T \in E(N, v) \backslash\{N\}$ and so $x^{S}(i)=v(\{i\})<y(i)$ for each $i \in S$, each $y \in \operatorname{LCS}(N, v)$ where $x^{C} \ll y$. The first part of the proof follows.

(b) Pick any $j \in C$ and define imputation $x$ as follows: $x(j)=v(N)$ and $x(i)=0$ for each $i \in N \backslash\{j\}$. By Theorem 5, imputation $x \in C(N, v)$ and by the first part of the proof, $x \notin L C S(N, v)$. This completes the proof of the second part of Theorem 6 .

We close this section with a characterization of the largest consistent set in big clan games. Let $(N, v)$ be a big clan game with big clan $C \in E(N, v)$. Denote by $j$ the only player outside the clan $C$ and define the subset of imputations $V(N, v)$ as

$$
V(N, v)=\{x \in I(N, v) \mid x(i)>0, i \in C, x(j) \geq v(N)-v(C)\} .
$$

Note that $x(j) \geq v(N)-v(C)$ implies $x(C) \leq v(C)$ and reciprocally. Theorem 7 below establishes that $V(N, v)$ constitutes the largest consistent set of such big clan games. Moreover, the union of all farsighted stable sets is equal to the largest consistent set. In case the marginal contribution of the 
powerless player $j$ is strictly positive, each imputation of $V(N, v)$ is strictly individually rational and assigns to player $j$ a payoff greater than or equal to his marginal contribution to coalition $N$. In contrast to imputations of the core, each imputation of the largest consistent set assigns to each player of the game a strictly positive payoff. In particular, the powerless player gets at least the best payoff he can expect in the core, that is, $v(N)-v(C)>0$. In case the marginal contribution of the powerless player is null, the largest consistent set is the set of imputations which assigns to each player of the clan a strictly positive payoff. Once again, the powerless player can expect more than his marginal contribution but cannot avoid situations where he gets a null payoff since $v(N)-v(C)=0$.

Theorem 7 Let $(N, v)$ be a big clan game with clan $C \in E(N, v)$. Then, (a) $F S(N, v)=\{\{x\} \mid x \in V(N, v)\}$ and (b) $L C S(N, v)=V(N, v)$.

Proof (a) Because $E(N, v) \supset\{N\}$, every farsighted stable set is a singleton $\{x\}$ where imputation $x$ is such that $(x, C) \in P(N, v)$ by Theorem 3. Pick any $x \in V(N, v)$. We have $x(i)>0$ for each player $i \in C$ and $x(C) \leq v(C)$ so that $(x, C) \in P(N, v)$ and thus $\{x\} \in F S(N, v)$. This proves that each element of $V(N, v)$ constitutes a farsighted stable set. It remains to show that no other imputation constitutes a farsighted stable set. Pick any $x \in I(N, v) \backslash V(N, v)$. If $x(C)>v(C)$, which is possible only if $v(N)-v(C)>0$, then $(x, C) \notin P(N, v)$ and so $\{x\}$ cannot be farsighted stable. If $x(C)=v(C)$ and $x(i)=0$ for some $i \in C$, then the same conclusion holds. Part (a) of Theorem 7 follows.

(b) Because the largest consistent set contains each farsighted stable set, the largest consistent set contains $V(N, v)$ by part (a). It remains to prove the no other imputation belongs to the largest consistent set. To this end, pick any $x \in I(N, v) \backslash V(N, v)$. If $x(i)=0$ for some $i \in C$, then imputation $x$ does not belong to the largest consistent by Theorem 6 . So, assume that $x(i)>0$ for each $i \in C$ and $0 \leq x(j)<v(N)-v(C)$, which is possible only if $v(N)-v(C)>0$. Pick any player $i_{1} \in C$ and define imputation $z$ as follows: $z\left(i_{1}\right)=v(N)$ and $z(i)=0$ for each other player $i$. Consider the move $x \longrightarrow\{j\} z$. By Theorem 6, $z$ does not belong to the largest consistent set. So, consider any $y \in \operatorname{LCS}(N, v)$ where $z \ll y$. By Fact $2,(y, C) \in P(N, v)$. This means that $y(i)>0$ for each $i \in C, y(C) \leq v(C)$ and so $y(j) \geq v(N)-v(C)$. Therefore, $x(j)<y(j)$. We have shown that $x$ satisfies condition (b) of the definition of the largest consistent set. Part (b) of Theorem 7 follows. 
Using the results obtained in Theorem 7, the next example illustrates a bankruptcy problem with one small claimant. It generalizes the one given in introduction.

Example 3 A bankruptcy game with one small claimant

Consider the bankruptcy problem $B=\left(N, e,\left(d_{i}\right)_{i \in N}\right)$ with claimant set $N=$ $\{1,2, \ldots, n\}$, estate $e>0$, claims $d_{i} \geq e$ for all $i \neq j$ and $d_{j}<e$. Then, the corresponding bankruptcy game $\left(N, v_{B}\right)$ is a big clan game with clan $C=N \backslash\{j\}$ and coalition function defined as $v_{B}(N)=e, v_{B}(C)=e-d_{j}$, and $v(S)=0$ for all remaining coalitions. Each member of the clan is a big claimant and $j \notin C$ is a small claimant. By Theorem 5, the core or equivalently the bargaining set of this game is given by

$$
C\left(N, v_{B}\right)=\left\{x \in I\left(N, v_{B}\right) \mid x(j) \leq d_{j}\right\}
$$

Because this bankruptcy game is supermodular, the core coincides with the unique stable set with respect to the relation $<$ and the Shapley value belongs to the core (see Shapley, 1971). The Shapley value allocates to each player a payoff strictly inferior to his claim, that is, $\operatorname{Sh}\left(N, v_{B}\right)(i)=\left(e-d_{j}\right) / n$ for each $i \in C$ and $\operatorname{Sh}\left(N, v_{B}\right)(j)=d_{j} / n$. It is worth noting that core imputations do not allocate more than $d_{j}$ units of money to the small claimant $j$. Furthermore, there exist core imputations which assign to some big claimants a null payoff. Assume now that the game is played by farsighted players. By Theorem 7 , the largest consistent set coincides with the collection of farsighted stable sets and is given by

$$
\operatorname{LCS}\left(N, v_{B}\right)=\bigcup_{x \in F S\left(N, v_{B}\right)}\{x\}=\left\{x \in I(N, v) \mid x(i)>0, i \in C, x(j) \geq d_{j}\right\} .
$$

The intersection between $C\left(N, v_{B}\right)$ and $\operatorname{LCS}\left(N, v_{B}\right)$ is given by

$$
\left\{x \in I(N, v) \mid x(i)>0, i \in C, x(C)=e-d_{j}, x(j)=d_{j}\right\} .
$$

We see that the core predicts very different outcomes compared with solutions which capture foresight of the players. In particular, each farsighted stable set corresponds to an imputation which assigns to each player of the clan a strictly positive payoff and to player $j$ a payoff greater than or equal to his claim. If, moreover, $d_{j}>e / 2$, then the small claimant gets a payoff strictly superior to one half of the estate and each player of the clan gets a strictly 
smaller payoff than $j$. A property of the largest consistent set is that any claim that exceeds the estate cannot be allocated to its claimant. So, the small claimant is the only one who gets his claim in the largest consistent set. To understand why the small claimant $j$ has a strict interest to induce a deviation from core imputations which assigns to him a payoff strictly inferior to $d_{j}$, consider, for example, the Shapley value and imputation $y^{k}$ defined as $y^{k}(i)=0$ for each $i \in C \backslash\{k\}, y^{k}(k)=e, k \neq j$, and $y(j)=$ 0 . If player $j$ deviates from the Shapley value to imputation $y^{k}$, he knows that members of $C \backslash\{k\}$ are strictly worse off and he or she anticipates that coalition $C \backslash\{k\}$ will make a counter offer. Coalition $C \backslash\{k\}$ is powerless and anticipates that player $k$ will not take seriously a proposal which assigns to him a null payoff. So, coalition $C \backslash\{k\}$ decides to allocate the total wealth to player $j$, knowing that player $k$ may, later on, make a counter proposal. Player $k$ has several alternatives but he knows that the clan $C$ is the only coalition which has the power to enforce any imputation, say $y$, where he gets a strictly positive payoff. In each move of the process initiated by player $j$ and leading to $y$, the active coalition strictly prefers the final outcome $y$ to the alternative it faces at that step. This implies that $y(i)>0$ for each $i \in C$ and $y(C) \leq e-d_{j}$ so that $y(j) \geq d_{j}$. The players are sufficiently clever to understand that the bargaining process will lead to such a final outcome $y$ which is farsighted stable. If players' expectations come to be focused on some particular farsighted stable outcome, they know that each other imputation is unstable with respect to $\ll$ because each farsighted stable set is a singleton set. These expectations will give them incentives to behave in such a way as to justify these expectations.

\section{Stability of the Shapley value}

When players are myopic, it is intuitively clear why it is desirable to use core allocation. However, there are two possible problems with this rule of thumb. The first one is that the core may be empty, even in superadditive TU-games. The second one is that the core may contain many elements. To get around this problem, several allocation rules have been introduced. An allocation rule is a function that assigns a unique allocation to each TU-game. A prevalent allocation rule in the literature is the Shapley value, introduced by Shapley (1953). There are several ways of explaining the Shapley value. For our purpose, it can be viewed as a procedure in which players decide 
to play the TU-game in the grand coalition. The latter is formed by adding players one at a time, where the order in which the players join is determined at random and all orders are equally likely. Each player gets the value he contributes to the coalition at the moment he joins it. The expected value to the players under this procedure is the Shapley value. Formally, the Shapley value is the map $S h$ from the set of essential TU-games $(N, v)$ into $\mathbb{R}^{N}$ whose value at any $i \in N$ is given by

$$
S h(N, v)(i)=\sum_{\substack{S \in 2^{N} \\ i \in S}} \frac{(|N|-|S|) !(|S|-1) !}{|N| !}[v(S)-v(S \backslash\{i\})] .
$$

For an arbitrary TU-game $(N, v)$, the Shapley value is an efficient allocation, that is, $\operatorname{Sh}(N, v)(N)=v(N)$, but need not be individually rational. Whenever the TU-game is superadditive, the Shapley value is an imputation but need not be in the core and hence can be directly dominated by another imputation. Shapley (1971) shows that the Shapley value of a supermodular TU-games is in the core so that the Shapley value is not dominated with respect to the relation $<$. In this section we prove that the Shapley value is always a stable imputation in superadditive TU-games in the following sense:

Theorem 8 Let $(N, v)$ be superadditive TU-game. Then, $S h(N, v) \in C(N, v)$ or $\{S h(N, v)\} \in F S(N, v)$.

Note that Theorem 8 is not a corollary of Lemma 3 since the Shapley value of a superadditive TU-game need not be interior. Theorem 8 is useful because if we know that the Shapley value is not an element of the core, then we can assert that it indirectly dominates each other imputation of the game. Equivalently, if we know that the Shapley value does not constitute a farsighted stable set, then we can assert that it belongs to the core. Note that Theorem 8 does not exclude the situation where the Shapley value both belongs to the core and constitutes a farsighted stable set. Because each stable set with respect to the direct dominance relation $<$ contains the core, we can reformulate Theorem 8 as follows: the Shapley value of a superadditive TU-game belongs to a stable set or it is a farsighted stable set.

To prove this result, we need a few more definitions and one Lemma. Let $(N, v)$ be a superadditive TU-game. A player $i \in N$ is called inessential for a coalition $S$, where $i \in S$, if $v(S)-v(S \backslash\{i\})=v(\{i\})$. We denote by $D(S)$ 
the set of inessential players for $S$. In case $v(S)-v(S \backslash\{i\})>v(\{i\})$, player $i$ is said to be essential for the coalition $S$. Player $i \in N$ is inessential in $(N, v)$ if he is inessential for each coalition $S$ he belongs to. Otherwise, player $i$ is said to be essential in $(N, v)$.

Lemma 4 Let $(N, v)$ be a superadditive TU-game. If player $j$ is inessential in $(N, v)$, then $S h(N, v)(i)=S h\left(N \backslash\{j\}, v_{\mid N \backslash\{j\}}\right)(i)$ for each $i \in N \backslash\{j\}$.

Proof. Consider any superadditive TU-game $(N, v)$ which contains at least one inessential player, say $j$. Then, for each coalition $S \subseteq N \backslash\{j\}$, the marginal contribution $v(S \cup\{j\})-v(S)$ of player $j$ when added to coalition $S$ is equal to $v(\{j\})$. Thus, for each nonempty coalition $S \subseteq N \backslash\{j\}$, each $i \in S$, it holds that

$$
v(S \cup\{j\})-v((S \cup\{j\}) \backslash\{i\})=v(S)-v(S \backslash\{i\}) .
$$

Now, compute the Shapley value of any player $i \neq j$ in $(N, v)$. Using (1), we obtain:

$$
\begin{aligned}
S h(N, v)(i)= & \sum_{\substack{S \subseteq N \backslash\{j\} \\
i \in S}} \frac{(|N|-|S|) !(|S|-1) !}{|N| !}[v(S)-v(S \backslash\{i\})] \\
& +\sum_{\substack{S \subseteq N \backslash\{j\} \\
i \in S}} \frac{(|N|-|S|-1) !|S| !}{|N| !}[v(S \cup\{j\})-v((S \cup\{j\}) \backslash\{i\})] \\
= & \sum_{S \subseteq N \backslash\{j\}} \frac{(|N|-|S|) !(|S|-1) !+(|N|-|S|-1) !|S| !}{\mid i \in S}[v(S)-v(S \backslash\{i\})] \\
= & \sum_{S \subseteq N \backslash\{j\}} \frac{(|N|-|S|-1) !(|S|-1) !}{(|N|-1) !}[v(S)-v(S \backslash\{i\})] \\
= & S h\left(N \backslash\{j\}, v_{\mid N \backslash\{j\})(i) .}\right.
\end{aligned}
$$

Corollary 1 Let $(N, v)$ be a superadditive TU-game. Then, each essential player in $(N, v)$ is essential in the subgame $\left(N^{e}, v_{\mid N^{e}}\right)$, where $N^{e}$ is the set of essential players in $(N, v)$. 
PROOF. Because each inessential player in $(N, v)$ remains inessential in each subgame of $(N, v), S h\left(N^{e}, v_{\mid N^{e}}\right)(i)=S h(N, v)(i)$ for each $i \in N^{e}$ by applying inductively Lemma 4. Since $v_{\mid N^{e}}(\{i\})=v(\{i\})$ for each $i \in N$ and $i \in N^{e}$ is essential in $(N, v)$, we get

$$
S h\left(N^{e}, v_{\mid N^{e}}\right)(i)=S h(N, v)(i)>v(\{i\})=v_{\mid N^{e}}(\{i\}) \quad \text { for each } i \in N^{e} .
$$

Because the superadditivity of $(N, v)$ is inherited by the subgame $\left(N^{e}, v_{\mid N^{e}}\right)$, we conclude that each $i \in N^{e}$ is essential in $\left(N^{e}, v_{\mid N^{e}}\right)$.

PRoOF OF THEOREM 8: Choose any superadditive TU-game $(N, v)$. If $N$ is the only coalition in $E(N, v)$, then the assertion holds because $C(N, v)=$ $I(N, v)$. Now, assume that $E(N, v) \supset\{N\}$. Note that $E(N, v) \neq \emptyset$ implies that $N^{e} \neq \emptyset$. If $N^{e}=N$, then $\operatorname{Sh}(N, v)(i)>v(\{i\})$ for each $i \in N$ and the result follows by Lemma 3. In case $N^{e} \subset N$, consider the subgame $\left(N^{e}, v_{\mid N^{e}}\right)$. By Corollary 1, each player $i \in N^{e}$ is essential in $\left(N^{e}, v_{\mid N^{e}}\right)$. It then follows that the Shapley value of $\left(N^{e}, v_{\mid N^{e}}\right)$ is an interior imputation and so $S h\left(N^{e}, v_{\mid N^{e}}\right) \in C\left(N^{e}, v_{\mid N^{e}}\right)$ or $\left\{S h\left(N^{e}, v_{\mid N^{e}}\right)\right\} \in F S\left(N^{e}, v_{\mid N^{e}}\right)$ by Lemma 3. If $\left\{S h\left(N^{e}, v_{\mid N^{e}}\right)\right\} \in F S\left(N^{e}, v_{\mid N^{e}}\right)$, then there exists $S \in$ $2^{N^{e}} \backslash\left\{N^{e}, \emptyset\right\}$ such that $\left(S h\left(N^{e}, v_{\mid N^{e}}\right), S\right) \in P\left(N^{e}, v_{\mid N^{e}}\right)$. Applying inductively Lemma 4 yields $S h\left(N^{e}, v_{\mid N^{e}}\right)(i)=S h(N, v)(i)$ for each $i \in N^{e}$ and so $S h\left(N^{e}, v_{\mid N^{e}}\right)\left(N^{e}\right)=S h(N, v)\left(N^{e}\right)$. We conclude that $(S h(N, v), S) \in$ $P(N, v)$ and so $\{S h(N, v)\} \in F S(N, v)$ by Theorem 3. Now, consider the alternative case where $S h\left(N^{e}, v_{\mid N^{e}}\right)$ belongs to $C\left(N^{e}, v_{\mid N^{e}}\right)$. For each $i \in$ $N \backslash N^{e}$, we have $S h(N, v)(i)=v(\{i\})$, and for each $i \in N^{e}, S h\left(N^{e}, v_{\mid N^{e}}\right)(i)=$ $S h(N, v)(i)$. Thus, for each $S \subseteq N^{e}$, each $T \subseteq N \backslash N^{e}$, it holds that $S h(N, v)(S)=S h\left(N^{e}, v_{\mid N^{e}}\right)(S) \geq v_{\mid N^{e}}(S)=v(S)$ and

$$
S h(N, v)(S \cup T)=S h(N, v)(S)+S h(N, v)(T) \geq v(S)+\sum_{i \in T} v(\{i\})=v(S \cup T) .
$$

This shows that $S h(N, v)$ is a core imputation of $(N, v)$. This completes the proof of Theorem 8 .

Theorem 8 does not answer the question whether or not the Shapley value belongs to the largest consistent set of a superadditive TU-game which does not satisfy the sufficient condition of Proposition 1. Example 3 partially answers this question by showing that the Shapley value can lie outside the 
largest consistent set. From this example, we provide a necessary and sufficient condition for a TU-game to have the Shapley value in the largest consistent set.

Theorem 9 Let $(N, v)$ be a superadditive TU-game. Then, $S h(N, v) \notin$ $L C S(N, v)$ if and only if $|E(N, v) \backslash\{N\}|=1$ and $D(N)=\emptyset$.

PROOF. See Appendix B.

Note that a big clan game with clan $C \in E(N, v)$ and $v(N)-v(C)>0$ satisfies the condition of Theorem 9 . So, in every such big clan game, the largest consistent set excludes the Shapley value.

\section{Conclusion}

We conclude the paper with some remarks and possible extensions. Troughout the paper we have considered the problem of sharing the value of the grand coalition. In superadditive games, the worth generated by the grand coalition is the highest among the coalition set. However, this may not be the case if the game is not superadditive. One way to deal with this problem is to divide the worth that can be achieved under the most favorable coalition. Our model is flexible enough to allow for that modification. Especially, Theorems 1 to 4 are robust to that change. Another way to tackle this problem is to consider the total worth generated by a coalition structure and to divide among the players the highest total worth that can be achieved among all coalition structures. The feasibility constraints must be changed accordingly. An imputation $x$ will be called feasible from another imputation through a coalition $S$ if $x(T) \leq v(T)$ for each coalition $T$ belonging to the coalition structure induced by $S$. More generally, we can also study games in partition function form (Thrall and Lucas, 1963).

As noted by Chwe (1994), the definition of the largest consistent set does not incorporate the idea of "best response" along the deviating path. In fact, coalitions will move to outcomes which are better than the status quo, not necessarily to the best outcomes. This point is illustrated by the bankruptcy game given in introduction of this paper. Some imputations $x$ for which $x(\{1,2,3\})$ is less than 3 belong to the largest consistent even if the clan $\{1,2,3\}$ will surely form to move to another imputation $y$ which directly 
dominates $x$ and where $y(\{1,2,3\})=3$. To amend this problem, Xue (1998) introduces some perfection in the indirect dominance process. In his paper, a player compares the status quo to the final outcomes but he also takes into account how, if at all, the final outcomes can be reached. To capture "perfect foresight", Xue assumes that players consider possible deviations along the paths that lead to final outcomes. Looking back at our bankruptcy problem, the clan will consider possible deviations in the last step of the indirect dominance path. When coalition $\{1,2,3\}$ is called upon to move, one of its best responses is to propose a farsighted stable imputation $y$, which directly dominates $x$ and where $y(\{1,2,3\})=3$ and not to propose a farsighted stable imputation $x$, where $x(\{1,2,3\})<3$. Mauleon and Vannetelbosch (2004) introduce another refinement of the largest consistent set based on the assumption that players are cautious. This refinement, the largest cautious consistent set, is successfully applied to coalition formation games. Refinements of these sorts can constitute another possibility to deal with foresight within the framework of cooperative games with transferable utility.

A

PROOF OF Proposition 1. Choose any superadditive TU-game $(N, v)$. If $C(N, v)=\emptyset$, we are done. Thus, assume that $C(N, v) \neq \emptyset$. When $(N, v)$ is such that $E(N, v)=\{N\}$, then $C(N, v)=\operatorname{LCS}(N, v)$ by part (a) of the proof of Theorem 1. It remains to prove that the assertion holds in case $E(N, v) \supset\{N\}$. By assumption, it holds that $\bigcap_{S \in E(N, v)} S=\emptyset$. Pick any $x \in C(N, v)$. To show: for each $z \in I(N, v)$, each $S \in 2^{N} \backslash\{\emptyset\}$ such that $x \longrightarrow_{S} z$, there exists $y \in L C S(N, v)$, where $y=z$ or $z \ll y$, such that $y(i) \leq x(i)$ for at least one $i \in S$.

Pick any $z \in I(N, v), S \in 2^{N} \backslash\{\emptyset\}$ such that $x \longrightarrow_{S} z$. We split the proof in two parts.

(a) $S=N$. By Fact 1 and Lemma 2, we can pick $(y, S) \in P(N, v)$ such that $z \ll y$. By Theorem 3, the singleton set $\{y\}$ is farsighted stable and so $y \in L C S(N, v)$. First, assume that $y \neq x$. Since $x$ and $y$ belong to $I(N, v)$, there is $i \in N$ such that $x(i)>y(i)$, as desired. Next, assume that $y=x$. The irreflexivity of $<$ on $I(N, v)$ yields $x(i) \nless x(i)$ for each $i \in N$.

(b) $S \neq N$. Recall that $x \in C(N, v)$ implies that $x(T) \geq v(T)$ for each $T \in 2^{N}$. Thus, we have to consider three types of moves $x \longrightarrow_{S} z$. 
(b.1) $x(S) \geq v(S)>\sum_{i \in S} v(\{i\})$. By Fact 1 and Lemma 2, we can pick $(y, S) \in P(N, v)$ such $z \ll y$. By Theorem 3, the singleton set $\{y\}$ is farsighted stable and so $y \in \operatorname{LCS}(N, v)$. Because $x(S) \geq v(S) \geq y(S)$, we conclude that $x(i) \geq y(i)$ for at least one $i \in S$.

(b.2) $x(S)>v(S)=\sum_{i \in S} v(\{i\})$. Pick any $T \in E(N, v), T \neq N$. Because $x(S)>\sum_{i \in S} v(\{i\})$, there is $j \in S$ such that $x(j)>v(\{j\})$. We consider three cases depending on the location of player $j$.

(b.2.1) Assume that $j \in S \cap T$. Choose any $\varepsilon \in] 0, e(x,\{j\})[$, where $e(x,\{j\})=x(j)-v(\{j\})$. Construct allocation $y$ as follows: $y(j)=v(\{j\})+\varepsilon$, $y(i)=v(\{i\})+\left[v(T)-\sum_{i \in T} v(\{i\})-\varepsilon\right] /(|T|-1)$ for each $i \in T \backslash\{j\}$, and $y(i)=v(\{i\})+\left[v(N)-v(T)-\sum_{i \in N \backslash T} v(\{i\})\right] /(|N|-|T|)$ for each $i \in N \backslash T$. By construction, $y \in I(N, v)$ and $(y, T) \in P(N, v)$. According to Theorem 3 , the singleton set $\{y\}$ is farsighted stable and so $y \in L C S(N, v)$. Because $\{y\}$ is a farsighted stable set, we have $z \ll y$. Finally, $y(j)<x(j)$ and $j \in S$ shows that at least one player in $S$ does not strictly prefer $y$ to $z$.

(b.2.2) Assume that $j \notin S \cap T$ and $T \neq N \backslash\{j\}$. Construct allocation $y$ as follows: $y(j)=x(j), y(i)=v(\{i\})+\left[v(T)-\sum_{i \in T} v(\{i\})\right] /|T|$ for each $i \in$ $T$, and $y(i)=v(\{i\})+\left[v(N)-v(T)-x(j)-\sum_{i \in N \backslash(T \cup\{j\})} v(\{i\})\right] /(|N|-|T|-1)$ for each $i \in N \backslash(T \cup\{j\})$. We want to show that $(y, T) \in P(N, v)$. Since $y(T)=v(T)$ and $y(i)>v(\{i\})$ for each $i \in T$, it is enough to verify that $y \in I(N, v)$. First, observe that $y(N)=v(N)$. Next, $x \in I(N, v)$ and $x(j)=y(j)$ imply that

$$
x(T)-y(T)=y(N \backslash(T \cup\{j\}))-x(N \backslash(T \cup\{j\}))
$$

From this last observation, we obtain

$$
\begin{aligned}
& v(N)-v(T)-x(j)-\sum_{i \in N \backslash(T \cup\{j\})} v(\{i\}) \\
= & y(N)-y(T)-y(j)-\sum_{i \in N \backslash(T \cup\{j\})} v(\{i\}) \\
\geq & y(N)-y(T)-y(j)-y(N \backslash(T \cup\{j\})) \\
= & 0 .
\end{aligned}
$$

The two equalities follow from the construction of $y$. The inequality follows from $x \in C(N, v)$ and equality (2). Indeed, $x \in C(N, v)$ implies that $x(T) \geq v(T)$ and $x(N \backslash(T \cup\{j\})) \geq v(N \backslash(T \cup\{j\}))$. It then follows that $y(N \backslash(T \cup\{j\})) \geq x(N \backslash(T \cup\{j\})) \geq v(N \backslash(T \cup\{j\}))$ by combining equality (2) with equality $v(T)=y(T)$. By superadditivity of $(N, v)$, it holds 
that $v(N \backslash(T \cup\{j\})) \geq \sum_{i \in N \backslash(T \cup\{j\})} v(\{i\})$. Therefore, $y(N \backslash(T \cup\{j\})) \geq$ $\sum_{i \in N \backslash(T \cup\{j\})} v(\{i\})$ showing the first inequality. From the last equality, we obtain $y(i) \geq v(\{i\})$ for each $i \in N \backslash(T \cup\{j\})$, completing the proof of $y \in I(N, v)$. Since $(y, T) \in P(N, v)$, we again apply Theorem 3 which leads to $z \ll y$ and $y \in L C S(N, v)$. Finally $x(j)=y(j)$ and $j \in S$ shows that at least one player in $S$ does strictly prefer $y$ to $x$.

(b.2.3) Assume that $j \notin T \cap S$ and $T=N \backslash\{j\}$. By assumption, $\bigcap_{T \in E(N, v)} T=\emptyset$. Thus, there exists $R \in E(N, v)$ such that $R \neq T, N$. If $j \in S \cap R$, then we apply (b.2.1.), otherwise, we apply (b.2.2). So, from any $z \in I(N, v)$ such that $x \longrightarrow_{S} z$, it is possible to construct an imputation $y \in L C S(N, v)$ such that $z \ll y$ and $y(i) \leq x(i)$ for at least one $i \in S$, as desired.

(b.3) $x(S)=v(S)=\sum_{i \in S} v(\{i\})$. Because $S \notin E(N, v)$ and $\bigcap_{T \in E(N, v)} T=$ $\emptyset$ by assumption, we can choose $T \in E(N, v)$ such that $S \nsubseteq T$. Pick any $j \in S$ such that $j \notin T$. Construct allocation $y$ as follows: $y(j)=v(\{j\})$, $y(i)=v(\{i\})+\left[v(T)-\sum_{i \in T} v(\{i\})\right] /|T|$ for each $i \in T$, and $y(i)=v(\{i\})+$ $\left[v(N)-v(T)-\sum_{i \in N \backslash T} v(\{i\})\right] /(|N|-|T|-1)$ for each $i \in N \backslash(T \cup\{j\})$. We see that $(y, T) \in P(N, v)$ so that an application of Theorem 3 yields $z \ll y$ and $y \in L C S(N, v)$. Again, $y(j)=x(j)$ shows that at least one player in $S$ does not strictly prefer $y$ to $x$.

By (b.1), (b.2) and (b.3), it follows that for any $S \neq N, z \in I(N, v)$ such that $x \longrightarrow_{S} z$, we can construct $y \in L C S(N, v)$ such that $z \ll y$ and $y(i) \leq x(i)$ for at least one $i \in S$. This result constitutes part (b) of the proof. By combining part (a) with part (b), we conclude that the core imputation $x$ belongs to the largest consistent set. Since $x$ was an arbitrarily element of $C(N, v)$, Proposition 1 follows.

\section{B}

Proof of TheOrem 9. $(\Longrightarrow)$ Assume that $|E(N, v) \backslash\{N\}|=1$ and $D(N)=$ $\emptyset$. Denote by $S$ the unique coalition in $E(N, v) \backslash\{N\}$. Firstly, note that $S=$ $N \backslash\{j\}$ for a unique player $j \in N$; otherwise $S \cup\{i\} \subset N$ and superadditivity of $v$ imply that $S \cup\{i\} \in E(N, v) \backslash\{N\}$ for each $i \in N \backslash S$. Secondly, each player of $N \backslash\{j\}$ is essential for that coalition, that is, $D(N \backslash\{j\})=\emptyset$; otherwise, $N \backslash\{i, j\} \subset N$ and $i \in D(N \backslash\{j\})$ imply that $N \backslash\{i, j\} \subset E(N, v) \backslash\{N\}$. Thirdly, $E(N, v)=\{N, N \backslash\{j\}\}$ and superadditivity of $v$ imply that $v(T)=$ $\sum_{i \in T} v(\{i\})$ for each $T \in 2^{N} \backslash E(N, v)$. Fourthly, from above and $D(N)=\emptyset$, 
it is easy to compute the payoff received by player $j$ under the Shapley allocation rule, i.e.,

$$
\operatorname{Sh}(N, v)(j)=\frac{(|N|-1) v(\{j\})}{|N|}+\frac{v(N)-v(N \backslash\{j\})}{|N|}
$$

and $S h(N, v)(j)>v(\{j\})$. To prove that $S h(N, v) \notin L C S(N, v)$, we generalize the procedure used in Example 3. Pick any player $i_{1} \in N \backslash\{j\}$ and define three imputations $x^{1}, x^{2}$ and $x^{3}$ as follows: $x^{1}\left(i_{1}\right)=v(N)-\sum_{i \in N \backslash\left\{i_{1}\right\}} v(\{i\})$, $x^{1}(i)=v(\{i\})$ for each $i \in N \backslash\left\{i_{1}\right\} ; x^{2}(j)=v(N)-\sum_{i \in N \backslash\{j\}} v(\{i\}), x^{2}(i)=$ $v(\{i\})$ for each $i \in N \backslash\{j\} ; x^{3}(j)=v(N)-v(N \backslash\{j\}), x^{3}(i)=v(\{i\})+$ $\left[v(N \backslash\{j\})-\sum_{i \in N \backslash\{j\}} v(\{i\})\right] /(|N|-1)$ for each $i \in N \backslash\{j\}$. We proceed in four steps. In Step 1, we show that any imputation which indirectly dominates another imputation of this TU-game is strictly individually rational for each member of the coalition $N \backslash\{j\}$ and is feasible for that coalition. Step 2 shows that $x^{2}$ does not lie in the largest consistent set of this TU-game which means that the largest consistent set is strictly included in the set of imputations. Step 3 makes use of Step 2 to show that $x^{1}$ is not included in the largest consistent set. In Step 4, we use Step 3 to show that the Shapley value does not belong to the largest consistent set.

Step 1: Consider any two distinct imputations $x$ and $y$ such that $x \ll$ $y$. By Fact 2, there exists a coalition $S \in 2^{N} \backslash\{N, \emptyset\}$ such that $(y, S) \in$ $P(N, v)$. The only candidate is the coalition $N \backslash\{j\}$ and we have $y(N \backslash\{j\}) \leq$ $v(N \backslash\{j\})$ and $v(\{i\})<y(\{i\})$ for each $i \in N \backslash\{j\}$. This shows the claim.

Step 2: We show that $x^{2}$ satisfies condition (b) of the definition of the largest consistent set. Consider the move $x^{2} \longrightarrow_{N \backslash\{j\}} x^{3}$, where $x^{3}(N \backslash\{j\})=$ $v(N \backslash\{j\})$. Because $\left(x^{3}, N \backslash\{j\}\right) \in P(N, v)$, imputation $x^{3} \in L C S(N, v)$ by an application of Theorem 3. But, $x^{2}(i)<x^{3}(i)$ for each $i \in N \backslash\{j\}$, as desired. Next, for each other imputation $y \in L C S(N, v) \backslash\left\{x^{3}\right\}$ such that $x^{3} \ll y$, it holds that $y(N \backslash\{j\}) \leq v(N \backslash\{j\})$ and $v(\{i\})<y(\{i\})$ for each $i \in N \backslash\{j\}$ by Step 1. Because $x^{2}(i)=v(\{i\})$ for each $i \in N \backslash\{j\}$, it follows that $x^{2}(i)<y(i)$ for each $i \in N \backslash\{j\}$, as desired.

Step 3: As in Step 2, we show that $x^{1}$ satisfies condition (b) of the definition of the largest consistent set. Pick any player $i_{2} \in N \backslash\left\{j, i_{1}\right\}$ and consider the move $x^{1} \longrightarrow\left\{i_{2}\right\} x^{2}$, where $x^{2}\left(\left\{i_{2}\right\}\right)=v\left(\left\{i_{2}\right\}\right)$ and $x^{2} \notin L C S(N, v)$ by 
Step 2. Thus, for all $y \in L C S(N, v)$, where $x^{2} \ll y$, it must be true that $x^{2}\left(i_{2}\right)<y\left(i_{2}\right)$. But, if $x^{2} \ll y$, then $y(N \backslash\{j\}) \leq v(N \backslash\{j\})$ and $v(\{i\})<y(\{i\})$ for each $i \in N \backslash\{j\}$ by Step 1. Because $x^{1}\left(i_{2}\right)=v\left(\left\{i_{2}\right\}\right)$, it follows that $x^{1}\left(i_{2}\right)<y\left(i_{2}\right)$, as desired.

Step 4: Consider the move $\operatorname{Sh}(N, v) \longrightarrow_{\{j\}} x^{1}$, where $x^{1}(j)=v(\{j\})$. We know that $x^{1} \notin L C S(N, v)$ by Step 3. To show that the Shapley value does not belong to the largest consistent set, consider any $y \in L C S(N, v)$ such that $x^{1} \ll y$. By Step 1, $x^{1} \ll y$ implies that $y(N \backslash\{j\}) \leq v(N \backslash\{j\})$ so that $y(j)=v(N)-y(N \backslash\{j\}) \geq v(N)-v(N \backslash\{j\})$. By (3), superadditivity of $v$ and $D(N) \neq \emptyset$, we get $S h(N, v)(j)<y(j)$. It follows that the Shapley value does not belong to the largest consistent set of $(N, v)$.

$(\Longleftarrow)$ By contraposition. Assume that $|E(N, v) \backslash\{N\}| \neq 1$ or $D(N) \neq \emptyset$. We have to show that $S h(N, v) \in L C S(N, v)$. Firstly, if $|E(N, v) \backslash\{N\}|=0$, then $\operatorname{LCS}(N, v)=I(N, v)$ by Theorem 1 and the Shapley value belongs trivially to the largest consistent set. Secondly, if $|E(N, v) \backslash\{N\}|=1$ and $D(N) \neq \emptyset$, then it is easy to verify that $D(N)=\{j\}$ and using the expression of $S h(N, v)(j)$, we see that $S h(N, v)(j)=v(\{j\})$. Thus, $S h(N, v)(N \backslash\{j\})=$ $v(N)-v(\{j\})=v(N \backslash\{j\})$. By the first part of the proof, we also know that $D(N \backslash\{j\})=\emptyset$ and hence $S h(N, v)(i)>v(\{i\})$ for each player $i \in N \backslash\{j\}$. Therefore, $(S h(N, v), N \backslash\{j\}) \in P(N, v)$ and by Theorem 3, the Shapley value constitutes a farsighted stable set which is sufficient to prove that it belongs to the largest consistent set. Thirdly, if $|E(N, v) \backslash\{N\}|>1$ and $\bigcap_{S \in E(N, v) \backslash\{N\}} S=\emptyset$, then $C(N, v) \in L C S(N, v)$ by Proposition 1 and $S h(N, v) \in C(N, v)$ or $\{S h(N, v)\} \in F S(N, v)$ by Theorem 8. Therefore, the Shapley value belongs to the largest consistent set. It remains to consider the case where $|E(N, v) \backslash\{N\}|>1$ and $\bigcap_{S \in E(N, v) \backslash\{N\}} S \neq \emptyset$ whatever the condition on $D(N)$. Pick any $z \in I(N, v)$ and $R \in 2^{N} \backslash\{\emptyset\}$ such that $S h(N, v) \longrightarrow_{R} z$. To show that the Shapley value belongs to the largest consistent set, we have to verify that there exists $y \in L C S(N, v)$, where $y=z$ or $z \ll y$, such that $y(i) \leq S h(N, v)(i)$ for at least one player $i \in R$. Pick $T \in E(N, v) \backslash\{N\}$ with $D(T)=\emptyset$. This leaves two possibilities.

(a) $T \cap R \neq \emptyset$. Pick any $j \in T \cap R$. Because $D(T)=\emptyset, S h(N, v)(j)>v(\{j\})$. Choose any $\varepsilon \in] 0, m[$ where $m$ is the minimal value between $e(S h(N, v),\{j\})$ and $v(T)-\sum_{i \in T} v(\{i\})$. Define imputation $y$ as follows: $y(j)=v(\{j\})+\varepsilon$, $y(i)=v(\{i\})+\left[v(T)-\sum_{i \in T} v(\{i\})-\varepsilon\right] /(|T|-1)$ for each $i \in T \backslash\{j\}$, and 
$y(i)=v(\{i\})+\left[v(N)-v(T)-\sum_{i \in N \backslash T} v(\{i\})\right] /(|N|-|T|)$ for each $i \in N \backslash T$. Observe that $(y, T) \in P(N, v)$ so that $\{y\} \in F S(N, v)$ by Theorem 3. It follows that $y \in L C S(N, v)$ and $z \ll y$. By construction, $y(j)<S h(N, v)(j)$ and so $y(i) \leq S h(N, v)(i)$ for at least one player $i \in R$.

(b) $T \cap R=\emptyset$. Because $\bigcap_{S \in E(N, v) \backslash\{N\}} S=\emptyset$, coalition $R \notin E(N, v)$ so that $v(R)=\sum_{i \in R} v(\{i\})$. Define imputation $y$ as follows: $y(i)=v(\{i\})$ for each $i \in R, y(i)=v(\{i\})+\left[v(T)-\sum_{i \in T} v(\{i\})\right] /|T|$ for each $i \in T$, and, in case $R \cup T \subset N, y(i)=v(\{i\})+\left[v(N)-v(T)-\sum_{i \in N \backslash T} v(\{i\})\right] /(|N|-|R|-|T|)$ for each $i \in N \backslash(R \cup T)$. Observe that $(y, T) \in P(N, v)$ so that $\{y\} \in$ $F S(N, v)$ by Theorem 3. Thus, $y \in L C S(N, v)$ and $z \ll y$. Because $y(i)=v(\{i\})$ for each $i \in R, y(i) \leq S h(N, v)(i)$ for each $i \in R$. In case $R \cup T=N$, imputation $y$ is defined as: $y(i)=v(\{i\})$ for each $i \in R$ and $y(i)=v(\{i\})+\left[v(N)-\sum_{i \in N} v(\{i\})\right] /|T|$ for each $i \in T$. It is possible to draw the same conclusion as above. Therefore, parts (a) and (b) prove that $\operatorname{Sh}(N, v) \in \operatorname{LCS}(N, v)$.

\section{References}

[1] Aumann R.J., Maschler M. (1964), "The Bargaining Set for Cooperative Games", In Advances of Game Theory, edited by M. Dresher, L.S. Shapley, A.W. Tucker, Princeton, Princeton University Press.

[2] Branzei R., Dimitrov D., Tijs S. (2006), "Convex Games versus Clan Games", Discussion paper No 2006-58, CentER, Tilburg University.

[3] Chwe M.S-Y. (1994), "Farsighted Coalitional Stability", Journal of Economic Theory 63:299-325.

[4] Davis M., Maschler M. (1965), "The Kernel of a Cooperative Game", Naval Research Logistic Quarterly 12:223-259.

[5] Diamantoudi E., Xue L. (2005), "Lucas' Counter Example Revisited", Discussion paper, Department of Economics, McGill University.

[6] Gillies D.B. (1953), "Some Theorems on n-Person Games", Ph.D. thesis, Princeton, New Jersey, Princeton University.

[7] Harsanyi J.C. (1974), "An Equilibrium Point Interpretation of Stable Sets and a Proposed Alternative Definition", Management Science 20:1472-1495. 
[8] Potters J., Poos R., Tijs S., Muto S. (1989), "Clan Games", Games and Economic Behavior 1:275-293.

[9] Mauleon A., Vannetelbosch V. (2004), "Farsightedness and Cautiousness in Coalition Formation Games with Positive Spillovers", Theory and Decision 56:291-324.

[10] Muto S., Nakayama S., Potters J.A., Tijs S. (1988), "On Big Boss Games", The Economic Studies Quarterly 39:303-321.

[11] O'Neill B. (1982), "A Problem of Right Arbitration from the Talmud", Mathematical Social Sciences 2:345-371.

[12] Rosenthal R.W. (1972), "Cooperative Games in Effective Form", Journal of Economic Theory 5:88-101.

[13] Schmeidler D. (1969), "The Nucleolus of a Characteristic Function Game", Siam Journal of Applied Mathematics 17:1163-1170.

[14] Shapley L.S. (1953), "A Value for $n$-Person Games", In volume 2 of Contribution to the Theory of Games, edited by H.W. Kuhn, A.W. Tucker. Annals of Mathematics Studies. Number 28. Princeton University Press, Princeton.

[15] Shapley L.S. (1971), "Cores of Convex Games", International Journal of Game Theory 1:11-26.

[16] Thrall R.M., Lucas W.F. (1963), " $n$-Person Games in Partition Function Form", Naval Research Logisitics Quarterly 10:281-298.

[17] von Neumann J., Morgenstern O. (1944), The Theory of Games and Economic Behaviour, 3rd ed, 1953, Princeton, Princeton Univ. Press, NJ.

[18] Xue L. (1998), "Coalitional Stability under Perfect Foresight", Economic Theory 11:603-627.

[19] Xue L. (1997), "Nonemptiness of the Largest Consistent Set", Journal of Economic Theory 73:453-459.

[20] Xue L., Diamantoudi E. (2003), "Farsighted Stability in Hedonic Games", Social Choice and Welfare 21:39-61. 


\begin{tabular}{|c|c|c|}
\hline Nr. & Author & Title \\
\hline $07-58$ & Sylvain Béal & PERCEPTRON VERSUS AUTOMATON\&8727; \\
\hline $07-57$ & $\begin{array}{l}\text { Sylvain Béal } \\
\text { Jacques Durieu } \\
\text { Philippe Solal }\end{array}$ & Farsighted Coalitional Stability in TU-games \\
\hline $07-56$ & $\begin{array}{l}\text { Alen Nosic } \\
\text { Martin Weber }\end{array}$ & $\begin{array}{l}\text { Determinants of Risk Taking Behavior: The role of } \\
\text { Risk Attitudes, Risk Perceptions and Beliefs }\end{array}$ \\
\hline $07-55$ & $\begin{array}{l}\text { Michael F. Meffert } \\
\text { Thomas Gschwend }\end{array}$ & $\begin{array}{l}\text { Strategic Voting under Proportional Representation } \\
\text { and Coalition Governments: A Simulation and } \\
\text { Laboratory Experiment }\end{array}$ \\
\hline $07-54$ & Klaus Fiedler & $\begin{array}{l}\text { Pseudocontingencies - A key paradigm for } \\
\text { understanding adaptive cognition }\end{array}$ \\
\hline $07-53$ & $\begin{array}{l}\text { Florian Kutzner } \\
\text { Peter Freytag } \\
\text { Tobias Vogel } \\
\text { Klaus Fiedler }\end{array}$ & $\begin{array}{l}\text { Base-rate neglect based on base-rates in } \\
\text { experience-based contingency learning }\end{array}$ \\
\hline $07-52$ & $\begin{array}{l}\text { Klaus Fiedler } \\
\text { Yaakov Kareev }\end{array}$ & $\begin{array}{l}\text { Implications and Ramifications of a Sample-Size } \\
\text { Approach to Intuition }\end{array}$ \\
\hline $07-51$ & Klaus Fiedler & $\begin{array}{l}\text { The Ultimate Sampling Dilemma in } \\
\text { Experience-Based Decision Making }\end{array}$ \\
\hline $07-50$ & $\begin{array}{l}\text { Jürgen Eichberger } \\
\text { David Kelsey }\end{array}$ & Ambiguity \\
\hline $07-49$ & Tri Vi Dang & Information Acquisition in Double Auctions \\
\hline $07-48$ & Clemens Kroneberg & $\begin{array}{l}\text { Wertrationalität und das Modell der } \\
\text { Frame-Selektion }\end{array}$ \\
\hline $07-47$ & $\begin{array}{l}\text { Dirk Simons } \\
\text { Nicole Zein }\end{array}$ & Audit market segmentation and audit quality \\
\hline $07-46$ & $\begin{array}{l}\text { Sina Borgsen } \\
\text { Martin Weber }\end{array}$ & $\begin{array}{l}\text { False Consensus and the Role of Ambiguity in } \\
\text { Predictions of Othersí Risky Preferences }\end{array}$ \\
\hline $07-45$ & $\begin{array}{l}\text { Martin Weber } \\
\text { Frank Welfens }\end{array}$ & $\begin{array}{l}\text { An Individual Level Analysis of the Disposition } \\
\text { Effect: Empirical and Experimental Evidence }\end{array}$ \\
\hline
\end{tabular}




\begin{tabular}{|c|c|c|}
\hline Nr. & Author & 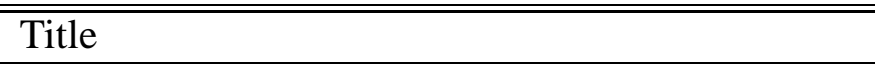 \\
\hline $07-44$ & $\begin{array}{l}\text { Martin Weber } \\
\text { Frank Welfens }\end{array}$ & $\begin{array}{l}\text { The Repurchase Behavior of Individual Investors: } \\
\text { An Experimental Investigation }\end{array}$ \\
\hline $07-43$ & $\begin{array}{l}\text { Manel Baucells } \\
\text { Martin Weber } \\
\text { Frank Welfens }\end{array}$ & $\begin{array}{l}\text { Reference Point Formation Over Time: A } \\
\text { Weighting Function Approach }\end{array}$ \\
\hline $07-42$ & $\begin{array}{l}\text { Martin Weber } \\
\text { Frank Welfens }\end{array}$ & $\begin{array}{l}\text { How do Markets React to Fundamental Shocks? Ar } \\
\text { Experimental Analysis on Underreaction and } \\
\text { Momentum }\end{array}$ \\
\hline $07-41$ & $\begin{array}{l}\text { Ernst Maug } \\
\text { Ingolf Dittmann }\end{array}$ & $\begin{array}{l}\text { Lower Salaries and No Options: The Optimal } \\
\text { Structure of Executive Pay }\end{array}$ \\
\hline $07-40$ & $\begin{array}{l}\text { Ernst Maug } \\
\text { Ingolf Dittmann } \\
\text { Christoph Schneider }\end{array}$ & Bankers and the Performance of German Firms \\
\hline $07-39$ & $\begin{array}{l}\text { Michael Ebert } \\
\text { Nicole Zein }\end{array}$ & $\begin{array}{l}\text { Wertorientierte Vergütung des Aufsichtsrats - } \\
\text { Auswirkungen auf den Unternehmenswert }\end{array}$ \\
\hline $07-38$ & $\begin{array}{l}\text { Ingolf Dittmann } \\
\text { Ernst Maug } \\
\text { Christoph Schneider }\end{array}$ & $\begin{array}{l}\text { How Preussag became TUI: Kissing too Many } \\
\text { Toads Can Make You a Toad }\end{array}$ \\
\hline $07-37$ & $\begin{array}{l}\text { Ingolf Dittmann } \\
\text { Ernst Maug }\end{array}$ & $\begin{array}{l}\text { Valuation Biases, Error Measures, and the } \\
\text { Conglomerate Discount }\end{array}$ \\
\hline $07-36$ & $\begin{array}{l}\text { Ingolf Dittmann } \\
\text { Ernst Maug } \\
\text { Oliver Spalt }\end{array}$ & $\begin{array}{l}\text { Executive Stock Options when Managers are } \\
\text { Loss-Averse }\end{array}$ \\
\hline $07-35$ & $\begin{array}{l}\text { Ernst Maug } \\
\text { Kristian Rydqvist }\end{array}$ & $\begin{array}{l}\text { Do Shareholders Vote Strategically? Voting } \\
\text { Behavior, Proposal Screening, and Majority Rules }\end{array}$ \\
\hline $07-34$ & $\begin{array}{l}\text { Ernst Maug } \\
\text { Abraham Ackerman }\end{array}$ & $\begin{array}{l}\text { Insider Trading Legislation and Acquisition } \\
\text { Announcements: Do Laws Matter? }\end{array}$ \\
\hline $07-33$ & Dirk Simons & Independence, low balling and learning effects \\
\hline $07-32$ & $\begin{array}{l}\text { Rainer Greifeneder } \\
\text { Herbert Bless }\end{array}$ & $\begin{array}{l}\text { Relying on accessible content versus accessibility } \\
\text { experiences: The case of processing capacity }\end{array}$ \\
\hline
\end{tabular}




\begin{tabular}{|c|c|c|}
\hline Nr. & Author & Title \\
\hline $07-31$ & $\begin{array}{l}\text { Rainer Greifeneder } \\
\text { Herbert Bless }\end{array}$ & $\begin{array}{l}\text { Depression and reliance on ease-of-retrieval } \\
\text { experiences }\end{array}$ \\
\hline $07-30$ & $\begin{array}{l}\text { Florian Heiss } \\
\text { Axel Börsch-Supan } \\
\text { Michael Hurd } \\
\text { David Wise }\end{array}$ & $\begin{array}{l}\text { Pathways to Disability: Predicting Health } \\
\text { Trajectories }\end{array}$ \\
\hline $07-29$ & $\begin{array}{l}\text { Axel Börsch-Supan } \\
\text { Alexander Ludwig } \\
\text { Mathias Sommer }\end{array}$ & Aging and Asset Prices \\
\hline $07-28$ & Axel Börsch-Supan & $\begin{array}{l}\text { GLOBAL AGING - Issues, Answers, More } \\
\text { Questions }\end{array}$ \\
\hline $07-27$ & Axel Börsch-Supan & $\begin{array}{l}\text { MIND THE GAP: THE EFFECTIVENESS OF } \\
\text { INCENTIVES TO BOOST RETIREMENT } \\
\text { SAVING IN EUROPE }\end{array}$ \\
\hline $07-26$ & Axel Börsch-Supan & Labor market effects of population aging \\
\hline $07-25$ & Axel Börsch-Supan & Rational Pension Reform \\
\hline $07-24$ & Axel Börsch-Supan & $\begin{array}{l}\text { European welfare state regimes and their generosity } \\
\text { towards the elderly }\end{array}$ \\
\hline $07-23$ & Axel Börsch-Supan & Work Disability, Health, and Incentive Effects \\
\hline $07-22$ & $\begin{array}{l}\text { Tobias Greitemeyer } \\
\text { Rainer Greifeneder }\end{array}$ & $\begin{array}{l}\text { Why the Euro looked like a price booster: } \\
\text { Differential perception of increasing versus } \\
\text { decreasing prices }\end{array}$ \\
\hline $07-21$ & $\begin{array}{l}\text { Patrick A. Müller } \\
\text { Rainer Greifeneder } \\
\text { Dagmar Stahlberg } \\
\text { Herbert Bless }\end{array}$ & $\begin{array}{l}\text { Relying on accessibility experiences in procedural } \\
\text { fairness judgments }\end{array}$ \\
\hline 07-20 & Volker Stocké & $\begin{array}{l}\text { The Motive for Status Maintenance and Inequality } \\
\text { in Educational Decisions. Which of the Parents } \\
\text { Defines the Reference Point? }\end{array}$ \\
\hline
\end{tabular}

